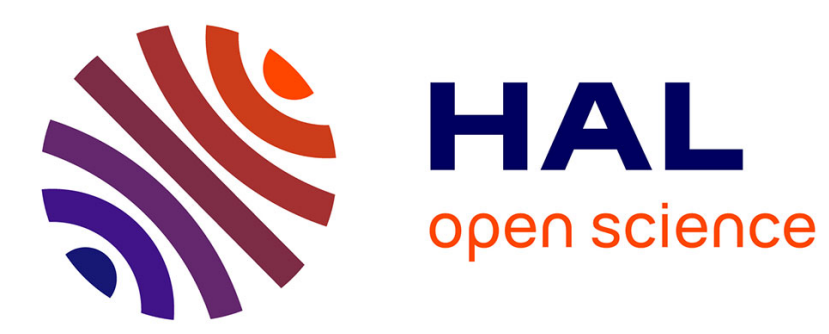

\title{
Review of Applications of Nonlinear Normal Modes for Vibrating Mechanical Systems
}

\author{
Konstantin Avramov, Yuri Mikhlin
}

\section{To cite this version:}

Konstantin Avramov, Yuri Mikhlin. Review of Applications of Nonlinear Normal Modes for Vibrating Mechanical Systems. Applied Mechanics Reviews, 2013, 10.1115/1.4023533 . hal-01335488

\section{HAL Id: hal-01335488 \\ https://hal.science/hal-01335488}

Submitted on 22 Jun 2016

HAL is a multi-disciplinary open access archive for the deposit and dissemination of scientific research documents, whether they are published or not. The documents may come from teaching and research institutions in France or abroad, or from public or private research centers.
L'archive ouverte pluridisciplinaire HAL, est destinée au dépôt et à la diffusion de documents scientifiques de niveau recherche, publiés ou non, émanant des établissements d'enseignement et de recherche français ou étrangers, des laboratoires publics ou privés. 


\title{
Review of Applications of Nonlinear Normal Modes for Vibrating Mechanical Systems
}

\author{
Konstantin V. Avramov \\ A. N. Podgorny Institute for Mechanical \\ Engineering Problems, \\ National Academy of Sciences of Ukraine, \\ Kharkov, Ukraine 61046; \\ National Technical University "KhPI," \\ Kharkov, Ukraine 61002 \\ e-mail: kvavr@kharkov.ua
}

Yuri V. Mikhlin

National Technical University "KhPl,"

Kharkov, Ukraine 61002

e-mail: muv@kpi.kharkov.ua

\begin{abstract}
This paper is an extension of the previous review, done by the same authors (Mikhlin, Y., and Avramov, K. V., 2010, "Nonlinear Normal Modes for Vibrating Mechanical Systems. Review of Theoretical Developments," ASME Appl. Mech. Rev., 63(6), p. 060802), and it is devoted to applications of nonlinear normal modes (NNMs) theory. NNMs are typical regimes of motions in wide classes of nonlinear mechanical systems. The significance of NNMs for mechanical engineering is determined by several important properties of these motions. Forced resonances motions of nonlinear systems occur close to NNMs. Nonlin-ear phenomena, such as nonlinear localization and transfer of energy, can be analyzed using NNMs. The NNMs analysis is an important step to study more complicated behavior of nonlinear mechanical systems.This review focuses on applications of Kauderer-Rosenberg and Shaw-Pierre concepts of nonlinear normal modes. The Kauderer-Rosenberg NNMs are applied for analysis of large amplitude dynamics of finite-degree-of-freedom nonlinear mechanical systems. Systems with cyclic symmetry, impact systems, mechanical systems with essentially nonlinear absorbers, and systems with nonlinear vibration isolation are studied using this concept. Applications of the Kauderer-Rosenberg NNMs for discretized structures are also discussed. The Shaw-Pierre NNMs are applied to analyze dynamics of finite-degree-of-freedom mechanical systems, such as floating offshore platforms, rotors, piece-wise linear systems. Studies of the Shaw-Pierre NNMs of beams, plates, and shallow shells are reviewed, too. Applications of Shaw-Pierre and King-Vakakis continuous nonlinear modes for beam structures are considered. Target energy transfer and localization of structures motions in light of NNMs theory are treated. Application of different asymptotic methods for NNMs analysis and NNMs based model reduction are reviewed.
\end{abstract}

\section{Introduction}

Nonlinear normal modes (NNMs) are a generalization of normal vibrations of linear systems. NNMs are used to detect properties of a nonlinear vibratory system, which are not obtained by other analytical and numerical methods. At first, the Kauderer-Rosenberg concept of NNMs, which is based on a construction of trajectories in the system configuration space, was developed to analyze large amplitude vibrations of finite-degreeof-freedom systems without dissipation. Later Shaw and Pierre proposed another concept of NNMs, based on the invariant manifolds theory. The Shaw-Pierre NNMs are used to investigate nonlinear dynamics of dissipative mechanical systems.

The Kauderer-Rosenberg NNMs are used to analyze nonlinear dynamics of systems with cyclic symmetry, impact systems, systems with essentially nonlinear absorbers, systems with nonlinear vibrations isolation, arches, beams, and cylindrical shells. The Shaw-Pierre NNMs are used to analyze nonlinear dynamics of floating offshore platform, double-tracked road vehicle, rotors, piece-wise linear systems, beams structures, plates and shells, and thin-walled structures interacting with fluids. A detailed review of theoretical investigations on NNMs is published in Ref. [1].

Continuous normal modes were developed from the 1990s. This approach is based on the direct solution of partial differential equations. King-Vakakis and Shaw-Pierre continuous normal modes are similar to the Kauderer-Rosenberg and Shaw-Pierre NNMs of finite-degree-of-freedom systems. Until now, it has been used to study nonlinear dynamics of beams structures only.
There are a lot of recent publications devoted to target energy transfer and localization of motions. These results are discussed in this review in the light of the NNMs theory.

Numerous studies used asymptotic methods to construct NNMs. Then modulation equations with respect to slow variables are derived; stability and bifurcations of NNMs are analyzed. Asymptotic methods were used to investigate massspring-pendulum systems, piece-wise linear two-degree-offreedom systems, one-dimensional continuous systems, beams structures, and water-filled circular shells.

Both theory and applications of NNMs to physical and engineering systems are treated in books [2-4]. Several aspects of the NNMs theory are considered in Refs. [5,6].

This paper gives a certain summary of NNMs applications. The main objectives of this review are to show how the NNMs methodology is used in mechanical engineering. The review is structured as follows. The Kauderer-Rosenberg NNMs and their applications are presented in Sec. 2. The Shaw-Pierre NNMs and their applications are treated in Sec. 3. NNMs of continuous systems are discussed in Sec. 4. A review of asymptotic methods for NNMs analysis is presented in Sec. 5. Target energy transfer and localization of motions in the light of the NNMs theory are treated in Sec. 6. NNMs based model reduction is discussed in Sec. 7. Finally, other references on NNMs are described in Sec. 8.

\section{Concept of Kauderer-Rosenberg NNMs and Its Applications}

2.1 Theory of the Kauderer-Rosenberg NNMs. Vibrations of thin-walled structures with geometrical nonlinearities are considered. Strains in such structures are small and displacements are 
moderate. Dynamics is described by partial differential equations, which has the following operator form $[4,7,8]$ :

$$
\begin{aligned}
& L(\Theta)+M(\Theta)+N(\Theta)=0 \\
& \left.B(\Theta)\right|_{\Theta \in \partial S}=0
\end{aligned}
$$

where $L(\Theta), M(\Theta)$ are operators of linear elastic and mass forces; $N(\Theta)$ is the nonlinear part of elastic forces acting on thin-walled structures; $\partial S$ is the thin-walled structure boundary, and $B(\Theta)$ is the operator of boundary conditions. For certain beam bending vibrations, the vector-function $\Theta$ consists of the lateral deflection of the beam $w(x, t)$ only, where $x$ is the longitudinal coordinate. To describe plates and shells dynamics lateral displacement $w(x, y, t)$ and the in-plane Airy stress function $\Phi(x, y, t)$ form the vector $\Theta$ otherwise, this vector is composed of the middle surface points displacements $(u(x, y, t) ; v(x, y, t) ; w(x, y, t))$.

Using the trial functions $\Psi_{k}(x, y)$, components of the vector $\Theta=\left(\Theta_{1}, \Theta_{2}, \ldots, \Theta_{s}\right)$ are expanded as

$$
\Theta_{s}=\sum_{k=1} r_{s k}(t) \Psi_{s k}(x, y)
$$

where $r_{s k}(t)$ are generalized coordinates of the mechanical system. One vector of $n$ components $q=\left(q_{1}, \ldots, q_{n}\right)$ is formed from these generalized coordinates. The Galerkin method is used to obtain the finite-degree-of-freedom model of thin-walled structure, which has the following vector form:

$$
\ddot{q}+F(q)=G(q, \dot{q}, t)
$$

where $F(q)=\left[F_{1}, \ldots, F_{n}\right]$ is the vector-function of nonlinear elastic forces; $F_{i}=\partial \Pi / \partial q_{i}$; $\Pi$ is the system potential energy, and vector-function $G(q, \dot{q}, t)$ is T-periodic.

A technique for NNMs calculations in configuration space is described in books [1-4]. Nonlinear vibrations of conservative systems are considered. Following Refs. [1-4], NNM is presented as single-valued functions of the generalized coordinate $q_{l}$ :

$$
q_{i}=q_{i}\left(q_{l}\right) ; \quad i=1, \ldots, l-1, l+1, \ldots, n
$$

The functions in Eq. (4) describe the Kauderer-Rosenberg NNMs. Curve $q_{i}\left(q_{l}\right)$ in the system configuration space is called a modal line. The generalized coordinates reach the maximal isoenergetic surface simultaneously in this mode. Such motions are described graphically by backbone curves. NNMs in Eq. (4) can be obtained from the following ordinary differential equations in a configuration space:

$$
2 q_{i}^{\prime \prime} \frac{h-\Pi}{1+\sum_{\substack{k=1 \\ k \neq l}}^{n} q_{k}^{\prime} 2}-q_{i}^{\prime} \Pi_{q_{l}}=-\Pi_{q_{i}}
$$

where $q_{i}^{\prime}=d q_{i} / d q_{l} ; \Pi_{q_{l}}=\partial \Pi / \partial q_{l} ; h$ is the value of the system energy. Equation (5) is derived using a new independent variable $q_{l}$ instead of $t$. Analytical continuation of the solutions up to the maximal isoenergetic surface $(h-\Pi=0)$ is possible if the following boundary conditions on this surface are added to Eq. (5) [1-4]:

$$
\begin{aligned}
& \Pi_{q_{l}}\left(q_{1}(Q), \ldots, q_{l-1}(Q), Q, q_{l+1}(Q), \ldots, q_{n}(Q)\right) q_{i}^{\prime}(Q) \\
& \quad=-\Pi_{q_{i}}\left(q_{1}(Q), \ldots, q_{l-1}(Q), Q, q_{l+1}(Q), \ldots, q_{n}(Q)\right)
\end{aligned}
$$

NNMs amplitudes $Q$ and the system energy $h$ satisfy the following equation:

$$
\Pi\left(q_{1}(Q), \ldots, q_{l-1}(Q), Q, q_{l+1}(Q), \ldots, q_{n}(Q)\right)=h
$$

A wide class of mechanical systems has potential energy in the following form: $\Pi=\Pi_{0}+\varepsilon \Pi_{1}$, where $\varepsilon$ is a small parameter. If $\varepsilon=0$, these dynamical systems have rectilinear modal lines $q_{i}=k q_{l}$. Then NNMs of perturbed systems can be obtained by the following asymptotic expansions [1-4]:

$$
q_{i}=k q_{l}+\sum_{\nu=1} \varepsilon^{\nu} q_{i \nu}\left(q_{l}\right), \quad q_{i \nu}=\sum_{r=1} a_{i \nu}^{(r)} q_{l}^{r}
$$

where $a_{i k}^{(r)}$ are unknown coefficients, which are determined.

2.2 NNMs of Finite-Degree-of-Freedom Systems. Free vibrations of a nonlinear system, which consists of $n$ equal nonlinear subsystems, are treated in Ref. [9]. These subsystems are coupled by weak nonlinear elastic elements $\varepsilon f_{i}^{(c)}$. Equations of such a system have the following form:

$$
\begin{aligned}
\ddot{q}_{i} & =f_{i}^{(u)}\left(q_{i}\right)+\varepsilon f_{i}^{(c)}\left(q_{i-1} ; q_{i} ; q_{i+1}\right) \\
i & =1, \ldots, n
\end{aligned}
$$

where $f_{i}^{(u)}$ is a restoring force of $i^{\text {th }}$ uncoupled subsystem. The system in Eq. (9) with cyclic symmetry is considered in Ref. [9]. As the unperturbed system in Eq. (9) $(\varepsilon=0)$ is uncoupled, NNMs are weakly curved. Therefore, the following expansion is true:

$$
q_{i}=\hat{q}_{i}\left(q_{1}\right)=\sum_{k=0} \varepsilon^{k} q_{i}^{(k)}\left(q_{1}\right) ; \quad i=2, \ldots, n
$$

These NNMs are determined from the system in Eq. (5) with the boundary conditions (Eq. (6)). It is shown that both localized and nonlocalized normal modes can be determined in a perfectly symmetric, weakly coupled structure. If the localized nonlinear mode is observed, vibrations amplitudes of one subsystem are essentially greater then the amplitudes of other subsystems. It is shown that, in the perfectly symmetric structure with weak coupling between the substructures, NNM localization exists (in contrast to the linear mode localization), and it does not depend on the existence of "mistuning" between substructures.

A two degree-of-freedom system with double-side impact constraints is treated in Ref. [10]. Differential equations (Eq. (5)) are used to calculate modal lines. Conditions of velocity instant jumps at impacts are added to these equations. NNMs are obtained for weak coupling between masses of the system. These motions are obtained in the form of asymptotic expansions by the coupling stiffness. It is obtained that the impact system possesses rich nonlinear behavior, including localized and nonlocalized periodic motions, as well as complicated bifurcation sequences, giving rise to new types of single- and double-side impacting motions, instabilities, and chaotic responses.

Lateral vibrations of a mass chain coupled by strings are considered in Ref. [11]. Every mass of the chain has nonlinear supports with impacts. The Kauderer-Rosenberg NNMs are used to obtain this system motions.

The Kauderer-Rosenberg NNMs are investigated in the selfsustained two-degree-of-freedom system [12]:

$$
\begin{aligned}
& \ddot{q}_{1}+q_{1}+\alpha q_{1}^{3}+\beta\left(q_{1}-q_{2}\right)-\varepsilon\left(1-q_{1}^{2}\right) \dot{q}_{1}=0 \\
& \ddot{q}_{2}+q_{2}+\alpha q_{2}^{3}+\beta\left(q_{2}-q_{1}\right)-\varepsilon \gamma\left(1-q_{2}^{2}\right) \dot{q}_{2}=0
\end{aligned}
$$

where $\alpha, \beta, \gamma$ are system parameters. Normal modes of selfsustained vibrations are analyzed in the configuration space. Periodic motions of forced viscoelastic nonlinear systems are considered in this paper, too.

An attempt to create a method for construction the forced system NNMs from experimental data is undertaken in Ref. [13]. The harmonic balance method is used to calculate NNMs numerically. 
The Kauderer-Rosenberg NNMs are used to analyze the absorption of mechanical vibrations by essential nonlinear attachment. A two-degree-of-freedom system consisting of the main one-degree-of-freedom subsystem and the essential nonlinear absorber is considered in Ref. [14]. Equations of motion for such system are the following:

$$
\begin{aligned}
& \varepsilon m \ddot{x}+c x^{3}+\gamma(x-y)=0 \\
& M \ddot{y}+k y+\gamma(y-x)=0
\end{aligned}
$$

where $y, x$ are generalized coordinates of the main linear subsystem and the nonlinear absorber, respectively. The mass of the absorber is essentially less than the mass of the main subsystem. This is indicated by the small parameter $\varepsilon \ll 1$. Both nonlocalized and localized NNMs exist in the system. Amplitudes of the main linear subsystem and the absorber are comparable in the nonlocalized NNM. The localized NNM is favorable for absorption of the linear subsystem vibrations, as vibrations amplitudes of the absorber and main subsystem are significant and small, respectively. The theoretical background from Sec. 2.1 is used to construct NNMs. Zero approximation by a small parameter of the nonlocalized NNM takes the form: $y_{0}=x+(c / \gamma) x^{3}$. Zero approximation by small parameter of the localized NNM takes the form: $y_{0}=0$. Power series in $\varepsilon$ are used to obtain NNMs. Ince algebraization is used to analyze the NNMs stability. It is shown that the nonlocalized NNM is unstable in the large range of system parameters and the localized NNM is stable for the most values of system parameters.

Similar procedures for calculations of the Kauderer-Rosenberg NNMs of the system with impact absorber and linear subsystem are proposed in Refs. [15,16]. The modal lines are calculated using NNMs theory and nonsmooth functions developed by Pilipchuk [6]. Ince algebraization permits to analyze NNMs stability.

Absorption of the linear subsystem by means of snap-through truss (Fig. 1), which jumps between three equilibrium positions, is analyzed in Ref. [17]. Equations of the system motions for shallow snap-through truss with respect to dimensionless variables and parameters are the following:

$$
\begin{aligned}
& \ddot{q}_{1}+(1+\varepsilon \bar{\gamma}) q_{1}-\frac{\varepsilon \bar{\gamma}}{\rho^{3}} q_{1} q_{2}^{2}-\frac{\varepsilon \bar{\gamma}}{2 \rho^{2}} q_{2}^{2}=0 \\
& \bar{\mu} \ddot{q}_{2}-\bar{\gamma} \alpha^{2} q_{2}-\frac{\bar{\gamma}}{\rho^{2}} q_{2} q_{1}+\frac{\bar{\gamma} \beta^{2}}{2} q_{2}^{3}=0
\end{aligned}
$$

where $\gamma, \rho, \alpha, \beta$ are system parameters. Nonlocalized and localized NNMs are obtained analytically.

The localized NNM takes the following form: $q_{1}=\varepsilon\left(a_{0}+a_{1} q_{2}\right.$ $\left.+a_{2} q_{2}^{2}+\cdots\right)$. This is a favorable motion for the vibration absorption, as main subsystem performs vibrations with small amplitudes $q_{1}=O(\varepsilon)$ and the absorber has moderate vibrations

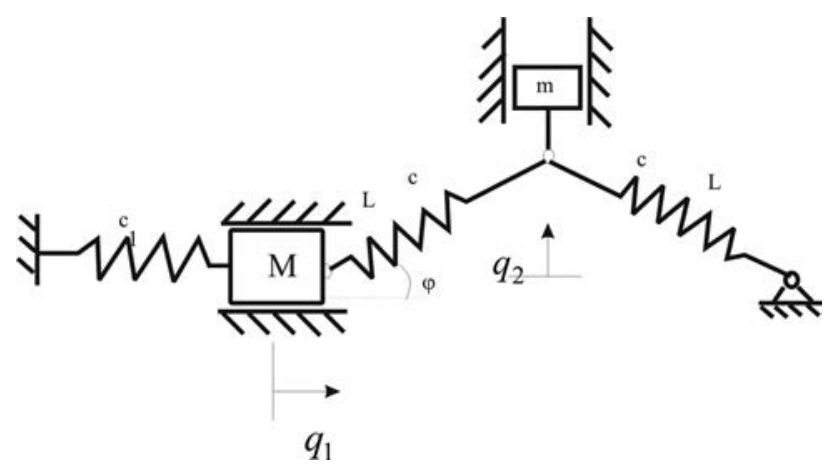

Fig. 1 Mechanical system, consisting of linear subsystem and snap-through truss. Adapted from Ref. [17]. amplitudes $q_{2}=O(1)$. Analysis of the stability shows that the localized NNM of the shallow snap-through truss is stable.

The forced vibrations of the system (Fig. 1) are examined in Refs. [18,19]. The external force $\varepsilon f \cos (\omega t)$ acts on the mass $M$ of the linear subsystem. Using dimensionless variables and simplifying nonlinear terms, the equations of motions become

$\ddot{q}_{1}+q_{1}=\varepsilon \bar{\gamma}\left\{-q_{1}+a_{1} q_{1}^{2}+a_{2} q_{2}^{2}+a_{3} q_{1} q_{2}^{2}+a_{4} q_{1}^{3}\right\}+\varepsilon f \cos (\omega t)$

$\ddot{q}_{2}+p^{2} q_{2}\left\{-a_{5}-a_{6} q_{1}-a_{7} q_{1}^{2}+a_{8} q_{2}^{2}\right\}=0$

where $a_{1}, \ldots, a_{8}$ are system parameters. Motions close to stable equilibrium are studied in Ref. [18].

Forced vibrations with large amplitudes of the snap-through truss are the most suitable for the vibration absorption [19]. Then the main subsystem performs vibrations with small amplitudes and the absorber has significant amplitudes of the vibrations: $q_{1}=O(\varepsilon) ; q_{2}=O(1)$. The unperturbed motions $(\varepsilon=0)$ satisfy the following equations:

$$
\ddot{q}_{2}+p^{2} q_{2}\left[-a_{5}+a_{8} q_{2}^{2}\right]=0 ; \quad q_{1} \equiv 0
$$

Jacobi elliptic functions are used to describe this mode. Modified Rauscher method is used to determine the forced NNM of the system in Eq. (13). Namely, the Eq. (14) solution takes the following form: $t=\left(\omega / p \sqrt{a_{5}}\right) F(\varphi, k)$, where $F(\varphi, k)$ is the elliptic integral of the second kind, $k$ is the integral modulus. This solution is substituted into the term $\varepsilon f \cos (\omega t)$ of the system in Eq. (13):

$$
\varepsilon f \cos (\omega t)=\varepsilon f g(\varphi)=\varepsilon f \cos \left[\frac{\omega}{p \sqrt{a_{5}}} F(\varphi, k)\right]
$$

Function $g(\varphi)$ is described by the truncated Fourier series:

$$
g(\varphi)=\cos \left[\frac{\pi F(\varphi, k)}{2 K(k)}\right] \approx A_{1} \cos \varphi+A_{3} \cos 3 \varphi+\cdots
$$

where $K(k)$ is the complete elliptic integral of the first kind. The expression in Eq. (16) is substituted into the right-hand part of the dynamical system of Eq. (13). As a result, the pseudoautonomous dynamical system is obtained and the Kauderer-Rosenberg NNM of this system is calculated. Then the iteration procedure is used to obtain a more precise NNM. Figure 2 shows the suitable NNM for the forced vibration absorption.

A three-degree-of-freedom nonlinear vibrations isolation system (Fig. 3) is considered in Ref. [20]. This system is described by the following ordinary differential equations:

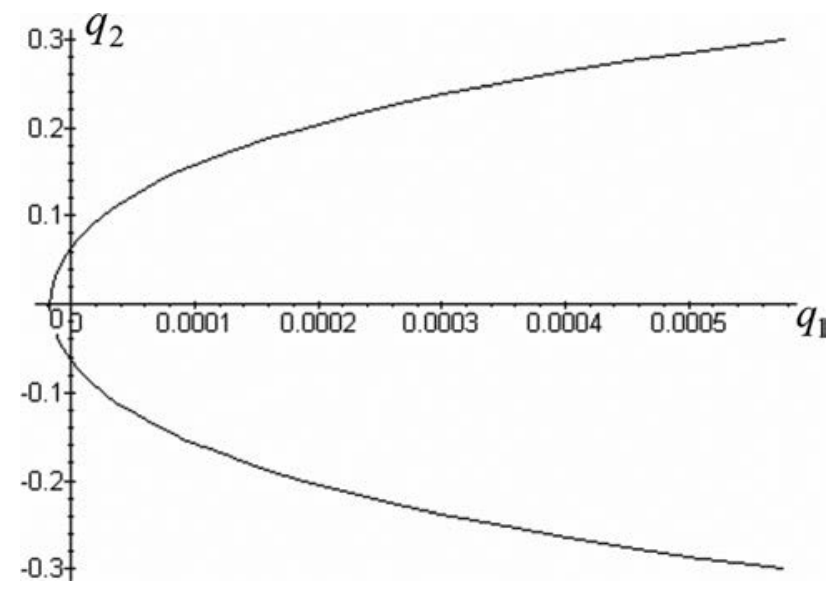

Fig. 2 Absorption motion. Adapted from Ref. [17]. 


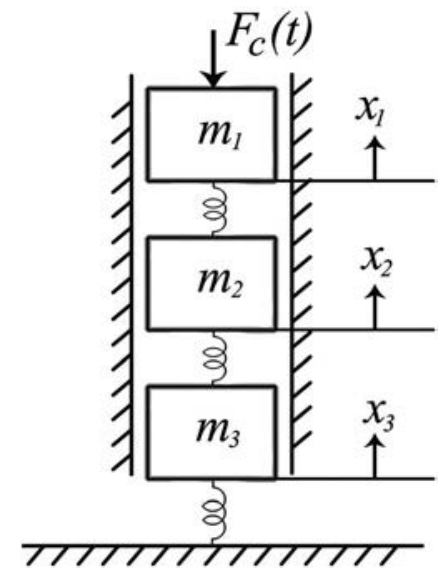

Fig. 3 Mechanical system with nonlinear vibrations isolation. Adapted from Ref. [20].

$$
\begin{aligned}
& m_{1} \ddot{x}_{1}+\varepsilon c_{1}\left(\dot{x}_{1}-\dot{x}_{2}\right)+f\left(x_{1}-x_{2}\right)=F_{C}(t) \\
& m_{2} \ddot{x}_{2}+\varepsilon c_{1}\left(\dot{x}_{2}-\dot{x}_{1}\right)+f\left(x_{2}-x_{1}\right)+\varepsilon k\left(x_{2}-x_{3}\right)=0 \\
& m_{3} \ddot{x}_{3}+\varepsilon c_{2}\left[\dot{x}_{3}-\dot{y}_{g}(t)\right]+g\left[x_{3}-y_{g}(t)\right]+\varepsilon k\left(x_{3}-x_{2}\right)=0
\end{aligned}
$$

where $f(\circ), g(\circ)$ are essential nonlinear elastic elements. Two external forces $F_{C}(t)$ and $y_{g}(t)$ act on the system. The machine, which is described by the generalized coordinates $x_{1}, x_{2}$, must be isolated from the external excitation $y_{g}(t)$. The system is designed to possess vibration isolation properties using strongly nonlinear stiffness elements. The system under consideration possesses localized NNMs whose energy is mainly confined in either one of its upper or lower parts. The Manevitch complexification technique is used in order to study the system dynamics. When this system is forced, the localized NNMs are excited by the periodic forcing and the steady state response also becomes localized. This results in vibration isolation of the part of the system which contains the least part of the steady state vibration energy.

Use of the nonlinear stiffness introduces new localized steady state responses which are beneficial for vibration isolation objectives. Localized steady responses are primarily responsible for fulfilling the dual mode vibration isolation objective.

\subsection{Kauderer-Rosenberg NNMs of Discretized Structure} Systems

2.3.1 Arch and Beam Systems. Vibrations of shallow arches are described by the following partial differential equations [21]:

$$
\frac{E A}{2 L} w_{x x} \int_{0}^{L}\left\{\left(w_{0, x}\right)^{2}-\left(w_{x}\right)^{2}\right\} d x+E I\left(w-w_{0}\right)_{x x x x}+\rho A w_{t t}=0
$$

where $w_{x x}=\partial^{2} w / \partial x^{2} ; w(x, t)$ is the arch deflection; $w_{0}(x)$ is initial imperfections; $A, I$ are area and moment of inertia of the cross section; $E, \rho$ are Young's modulus and material density, and $L$ is arch length. Arch deflection is expanded by using eigenmodes of a simply supported beam, $\sin (i(\pi x / L)) ; i=1,2, \ldots$. The finitedegree-of-freedom model of the arch dynamics is obtained in the following form:

$$
\begin{aligned}
\ddot{q}_{1}+\varepsilon q_{1} & =\left(1+q_{1}\right) \chi\left(q_{1}, q_{2}, \ldots\right) \\
\ddot{q}_{i}+\varepsilon i^{4} q_{i} & =i^{2} q_{i} \chi\left(q_{1}, q_{2}, \ldots\right) ; i=2,3, \ldots \\
\chi\left(q_{1}, q_{2}, \ldots\right) & \left.=-0.5 \quad q_{1}+0.5 \sum_{i=1}^{n} i^{2} q_{i}^{2}\right)
\end{aligned}
$$

where $q_{i}(t)$ are generalized coordinates of the system. Using the two first eigenmodes of bending vibrations and shifting the origin,

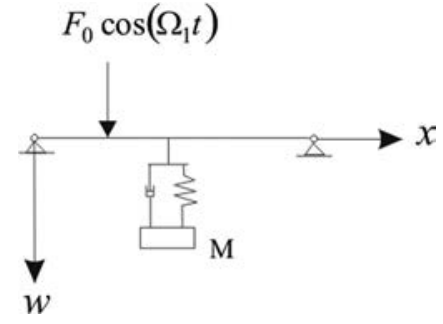

Fig. 4 The beam with nonlinear passive vibration absorber. Adapted from Ref. [22].

the system in Eq. (18) can be reduced to the following twodegree-of-freedom dynamical system:

$$
\begin{aligned}
\ddot{q}_{1}+\varepsilon\left(q_{1}-1\right) & =\theta_{1} \tilde{\chi}\left(q_{1}, q_{2}\right) \\
\ddot{q}_{2}+16 \varepsilon q_{2} & =4 q_{2} \tilde{\chi}\left(q_{1}, q_{2}\right) \\
\tilde{\chi}\left(q_{1}, q_{2}\right) & =-0.5\left[0.5\left(q_{1}^{2}-1\right)+2 q_{2}^{2}\right]
\end{aligned}
$$

Two NNMs of the system in Eq. (19) are investigated in Ref. [21]. The first NNM is described by weakly curved modal lines:

$$
q_{1}=\varepsilon g\left(q_{2}\right)=\varepsilon \sum_{i=0} b_{i} q_{2}^{i}
$$

where $b_{i}$ are parameters, which are calculated. If the trajectory in Eq. (20) is obtained, motions on NNM are determined by the following system:

$$
\ddot{q}_{2}+\varepsilon 16 q_{2}+\varepsilon^{2} q_{2} g^{2}\left(q_{2}\right)-q_{2}+4 q_{2}^{3}=0
$$

Equation (21) describes the system motions between three equilibriums. The harmonic balance method is used to obtain periodic motions.

The second NNM of the system in Eq. (19) is the following: $q_{2} \equiv 0$. The harmonic balance method is used to construct these motions. The Ince algebraization and the Hill determinants method are applied to analyze the NNMs stability in Refs. [2-4].

Vibrations of the beam interacting with essential nonlinear absorber (Fig. 4) are considered in Ref. [22]. System motions are described by the following equations with respect to the dimensionless variables and parameters:

$$
\begin{aligned}
& r^{2}\left(w_{t t}+w_{x x x x}\right)= 0.5 w_{x x} \int_{0}^{1} w_{x}^{2} d x+F_{0} \delta\left(x-\frac{1}{3}\right) \cos (\Omega t) \\
&+\delta\left(x-\frac{1}{2}\right) K\left[u-w\left(\frac{1}{2}, t\right)\right]^{3} \\
& \bar{M} r^{2} \ddot{u}+K\left[u-w\left(\frac{1}{2}, t\right)\right]^{3}=0
\end{aligned}
$$

where $w(x, t)$ is beam bending vibrations; $u(t)$ is the generalized coordinate of the absorber; $\delta(x)$ is the $\delta$ function, and $r$ is the radius gyration of the cross section. The absorber is attached in the middle of the beam. The external force acts at the point $x=0.5$. Two modes are used to describe the beam vibrations: $w(x, t)=r^{2}\left[q_{1}(t) \sin (\pi x)+q_{2}(t) \sin (2 \pi x)\right]$. Using the Galerkin method, the following three degree-of-freedom dynamical system is derived:

$\ddot{q}_{1}+\pi^{4} q_{1}+\varepsilon 0.25 \pi^{4}\left(q_{1}^{2}+4 q_{2}^{2}\right) q_{1}=\varepsilon f_{0} \cos (\Omega t)+\varepsilon 2 K\left(\theta-q_{1}\right)^{3}$

$\ddot{q}_{2}+16 \pi^{4} q_{2}+\varepsilon \pi^{4}\left(q_{1}^{2}+4 q_{2}^{2}\right) q_{2}=\varepsilon f_{0} \cos (\Omega t)$

$M \ddot{\theta}+K\left(\theta-q_{1}\right)^{3}=0$ 
where $\theta$ is the dimensionless generalized coordinate of the absorber. The combination of NNMs and Rauscher methods is used to analyze the nonlinear vibrations. In zero approximation ( $\varepsilon=0$ ), it is assumed that $q_{1}=q_{2}=0$ and the motions of the absorber take the form:

$$
\theta=\theta_{\max } c n\left(\sqrt{2} \theta_{\max } \chi t, k\right)=\theta_{\max } \cos \varphi
$$

where $c n\left(\sqrt{2} \theta_{\max } \chi t, k\right)$ is the Jacobi function; $\theta_{\max }$ is the vibrations amplitude; $\chi=\sqrt{K /(2 M)}$. Equation (24) is inverted and it is obtained that

$$
\cos (\Omega t)=\cos \left[\frac{\Omega}{\sqrt{2} \theta_{\max } \chi} F(\varphi, k)\right]=A_{1} \cos \varphi+A_{3} \cos 3 \varphi+\cdots
$$

Equation (25) is substituted into the right-hand part of the system in Eq. (23) and the pseudoautonomous dynamical system is obtained. Using the iteration procedure, NNMs of the forced vibrations are obtained. As follows from the numerical simulation, the localized NNM takes place in a wide band of the excitation frequency.

Bending vibrations of the cantilever beam with a transverse edge crack are studied in Ref. [23]. With nonlinear boundary conditions associated with the crack being open or closed, by modeling the cracked beam as two linear configurations (open crack and closed crack) the piecewise-linear two-degree-of-freedom system is obtained. This system takes the following form:

$$
\begin{aligned}
& m_{1} \ddot{x}_{1}+\left[k_{1}+k_{3}+k_{2} H\left(x_{1}\right)\right] x_{1}-k_{3} x_{2}=0 \\
& m_{2} \ddot{x}_{2}-k_{3} x_{1}+k_{3} x_{2}=0
\end{aligned}
$$

where

$$
H\left(x_{1}\right)=\left\{\begin{array}{l}
1 ; x_{1}<0 \\
0 ; x_{1}>0
\end{array}\right.
$$

Spring $k_{2}$ has small stiffness: $k_{2}=\varepsilon k$. In region $x_{1}>0$, modal coordinates are used and the system in Eq. (26) takes the following form: $\ddot{x}+\omega_{11}^{2} x=0 ; \ddot{y}+\omega_{12}^{2} y=0$. For $x_{1}<0$, the system in Eq. (26) has the following form:

$$
\begin{aligned}
& \ddot{x}=-\left(\omega_{11}^{2}+a_{1}^{2} k_{2}\right) x-a_{1} a_{2} k_{2} y \\
& \ddot{y}=-a_{1} a_{2} k_{2} x-\left(\omega_{12}^{2}+a_{2}^{2} k_{2}\right) y
\end{aligned}
$$

NNM of the system in Eq. (26) is a small deviation from the line $y=0$. Therefore, this NNM can be presented as

$$
y(x)=\varepsilon \begin{cases}f_{1}(x) ; & x_{1}>0 \\ f_{2}(x) ; & x_{1}<0\end{cases}
$$

Equations with respect to $f_{1}(x)$ and $f_{2}(x)$ are derived from the system in Eq. (26):

$$
\begin{aligned}
& \left(2 h-\omega_{11}^{2} x^{2}\right) f_{1}^{\prime \prime}-\omega_{11}^{2} x f_{1}^{\prime}+\omega_{12}^{2} f_{1}=0+O(\varepsilon) \\
& \left(2 h-\omega_{11}^{2} x^{2}\right) f_{2}^{\prime \prime}-\omega_{11}^{2} x f_{2}^{\prime}+\omega_{12}^{2} f_{2}=-k a_{1} a_{2} x+O(\varepsilon)
\end{aligned}
$$

NNM in configuration space is obtained from Eq. (27).

2.3.2 Cylindrical Shells. Thin, simply supported cylindrical shells are considered. For such shells, frequencies of longitudinal vibrations are significantly higher than frequencies of transverse motions. Therefore, the longitudinal inertia and shear are not taken into account. Shells with geometrical nonlinearity are considered; in this case, strains are small and displacements are moderate. Standing and traveling waves take place in closed cylindrical shells. The Kauderer-Rosenberg NNMs are successfully used to analyze large amplitude vibrations of the cylindrical shells. Vibrations of shells with geometrical nonlinearity are described by the Donnell equations [24]:

$$
\begin{aligned}
& \frac{D}{h} \nabla^{4} w_{1}=L\left(w_{1}+w_{0}, \Phi\right)+\frac{1}{R} \frac{\partial^{2} \Phi}{\partial x^{2}}-\rho \frac{\partial^{2} w_{1}}{\partial t^{2}} \\
& \frac{1}{E} \nabla^{4} \Phi=-\frac{1}{2} L\left(w_{1}+2 w_{0}, w_{1}\right)-\frac{1}{R} \frac{\partial^{2} w_{1}}{\partial x^{2}}
\end{aligned}
$$

where $L(A, B)=\left(\partial^{2} A / \partial x^{2}\right)\left(\partial^{2} B / \partial y^{2}\right)+\left(\partial^{2} A / \partial y^{2}\right)\left(\partial^{2} B / \partial x^{2}\right)$ $-2\left(\partial^{2} A / \partial x \partial y\right)\left(\partial^{2} B / \partial x \partial y\right) ; D=E h^{3} / 12\left(1-\mu^{2}\right) ; E$ is the Young modulus; $\boldsymbol{\mu}$ is the Poisson ratio; $\boldsymbol{\rho}$ is the shell material density; $y, x=R \theta$ are longitudinal and circumference coordinates; $w_{1}$ is the radial displacements of the middle surface; $\Phi$ is the in-plane Airy stress function, and $w_{0}$ is initial imperfections of the shell.

A two-degree-of-freedom nonlinear system, which is obtained using the Galerkin method, is treated using the NNMs approach in Ref. [2]. Dependences of the vibration frequencies on amplitudes are obtained.

Vibrations of the cylindrical shells with geometrical nonlinearity and initial imperfections are considered in Refs. [25,26]. Initial imperfections have the following form:

$$
w_{0}=f_{10} \sin n \theta \sin \left(\frac{m \pi y}{L}\right)+f_{20} \sin n \theta \sin \left(\frac{m \pi y}{L}\right)
$$

where $n$ is a number of waves in the circumference direction; $m$ is a number of half-wave in the longitudinal direction; $f_{10}, f_{20}$ are amplitudes of the initial imperfections. The shell radial deflections are approximated as

$$
\begin{aligned}
w_{1}= & f_{1}(t) \sin n \theta \sin \left(\frac{m \pi y}{L}\right)+f_{2}(t) \sin n \theta \sin \left(\frac{m \pi y}{L}\right) \\
& +f_{3}(t) \sin ^{2}\left(\frac{m \pi y}{L}\right)
\end{aligned}
$$

It is assumed that length of the middle surface transverse section is constant

$$
\left.\int_{0}^{2 \pi R}-\frac{w_{1}}{R}+\frac{1}{2}\left(\frac{\partial w_{1}}{\partial y}\right)^{2}\right) d y=0
$$

Equation (31) is reduced into the following relation: $f_{3}=\left(n^{2} / 4 R\right)$ $\left(f_{1}^{2}+f_{2}^{2}\right)$. The solution in Eq. (30) is substituted into the second equation of the system in Eq. (28) and the Airy stress function is calculated. Applying the Galerkin procedure to the first equation of the system in Eq. (28), a two-degree-of-freedom nonlinear dynamical system is derived [27]:

$$
\begin{aligned}
& \ddot{f}_{1}+\omega_{1}^{2} f_{1}+\gamma f_{2}+2 \chi f_{1}\left(\dot{f}_{1}^{2}+f_{1} \ddot{f}_{1}+\dot{f}_{2}^{2}+f_{2} \ddot{f}_{2}\right)+\gamma_{1} f_{1}\left(f_{1}^{2}+f_{2}^{2}\right) \\
& \quad+\alpha_{1} f_{1} f_{2}+\alpha_{2} f_{1}^{2}+\alpha_{3} f_{2}^{2}=\omega_{0}^{2} f_{10} \\
& \ddot{f}_{2}+\omega_{2}^{2} f_{2}+\gamma f_{1}+2 \chi f_{2}\left(\dot{f}_{1}^{2}+f_{1} \ddot{f}_{1}+\dot{f}_{2}^{2}+f_{2} \ddot{f}_{2}\right)+\gamma_{1} f_{2}\left(f_{1}^{2}+f_{2}^{2}\right) \\
& \quad+\beta_{3} f_{1} f_{2}+\beta_{1} f_{1}^{2}+\beta_{2} f_{2}^{2}=\omega_{0}^{2} f_{20}
\end{aligned}
$$

where $\omega_{0}, \omega_{i}, \gamma, \chi, \gamma_{1}, \alpha_{i}, \beta_{i}$ are the system parameters. As follows from Refs. [25,26], the modal line of the system in Eq. (32) is close to the rectilinear approximation $f_{2}=k f_{1}$. Parameter $k$ is determined by the initial imperfections: $k=f_{20} / f_{10}$. Obtained NNM is stable and verified by numerical simulation of the system.

Eigenfrequencies of the conjugate modes are close for a wide class of cylindrical shells. Interaction of eigenmodes with close frequencies is investigated in Refs. [28,29]. Donnell equations with respect to displacements are used to analyze shell vibrations. Shell radial deflections are expanded on $N_{M}$ eigenmodes as: 


$$
\begin{aligned}
w_{1}(x, \theta, t)= & \sum_{j=1}^{N_{M}}\left(w_{j}^{(C)}(t) \cos n_{j} \theta+w_{j}^{(S)}(t) \sin n_{j} \theta\right) \sin \left(\frac{\pi y}{L}\right) \\
& +w_{0}(t) \sin ^{4}\left(\frac{\pi y}{L}\right)
\end{aligned}
$$

where $w_{j}^{(c)}, w_{j}^{(s)}, w_{0}$ are shell generalized coordinates. Since frequency of the trial function $\sin ^{4}(\pi y / L)$ is significantly higher than the eigenfrequencies of the eigenmodes $\cos n_{j} \theta \sin (\pi y / L)$ and $\sin n_{j} \theta \sin (\pi y / L)$, coordinate $w_{0}(t)$ is derived in the form of the function of generalized coordinates $w_{j}^{(c)}, w_{j}^{(s)}$. Shell vibrations are described by the following finite-degree-of-freedom dynamical system:

$\ddot{w}_{i}^{(C)}+\omega_{i}^{2} w_{i}^{(C)}+w_{i}^{(C)} R_{i}\left(w^{(S)}, w^{(C)}\right)+G_{i}^{(C)}\left(w^{(S)}, w^{(C)}\right)=0$

$\ddot{w}_{i}^{(S)}+\omega_{i}^{2} w_{i}^{(S)}+w_{i}^{(S)} R_{i}\left(w^{(S)}, w^{(C)}\right)+G_{i}^{(S)}\left(w^{(S)}, w^{(C)}\right)=0$

$i=1, \ldots, N_{M}$

where $R_{i}, G_{i}^{(C)}, G_{i}^{(S)}$ are functions of the generalized coordinates. As a result of the symmetry in the system in Eq. (34), two NNMs are observed:

$$
w_{i}^{(S)}= \pm w_{i}^{(C)} ; \quad i=1, \ldots, N_{M}
$$

The nonlinear modes in Eq. (35) describe standing waves of cylindrical shells. Analysis of backbone curves of these NNMs is presented in Ref. [28].

Parametric vibrations of cylindrical shells under the action of longitudinal force $N_{x}(t)=N_{0}+N_{1} \cos (2 \nu t)$ are treated in Refs. [30,31]. Vibrations of these shells are described by the Donnell equations (Eq. (27)). Dynamic deflection takes the following form:

$$
\begin{aligned}
w_{1}= & \sum_{i=1}^{3}\left(q_{2 i-1} \cos n_{i} \theta+q_{2 i} \sin n_{i} \theta\right) \sin \left(\frac{m \pi y}{L}\right) \\
& +q_{7} \sin ^{2}\left(\frac{m \pi y}{L}\right)+q_{8}
\end{aligned}
$$

Generalized coordinates $q_{7}$ and $q_{8}$ are determined from quasistatic analysis [30,31]. Then the vibrations are described by the following six-degree-of-freedom nonlinear dynamical system:

$$
\begin{aligned}
& \ddot{q}_{i}+\omega_{i}^{2} q_{i}+q_{i} R_{i}\left(q_{1}, \ldots, q_{6}\right)+G_{i}\left(q_{1}, \ldots, q_{6}\right) \\
& \quad+\chi_{i} q_{i} N_{x}=0 ; \quad i=1, \ldots, 6
\end{aligned}
$$

where $R_{i}$ is a quadratic form of the generalized coordinates; $G_{i}$ are cubic functions with respect to the generalized coordinates, and $\chi_{i}$ are the system parameters. NNMs of the dynamical system in Eq. (37) are the following:

$$
q_{2 i-1}= \pm q_{2 i} ; \quad i=1,2,3
$$

These NNMs describe standing waves of the cylindrical shell [30]. Nonlinear modes, which are straight lines in a configuration space, are obtained for the multimode shells dynamics. The same NNMs exist both in the system without damping and in the damping system.

Nonlinear modes and traveling waves are two types of the shell motions. Normal modes and traveling waves coexist in the dimensionless frequency bands $\nu \in[1 ; 1.6]$ and $\nu \in[1.1 ; 1.8]$. Any one of these motions has a basin of attraction. Therefore, if the initial conditions belong to the basin of attraction of nonlinear mode or traveling waves, then the motion tends to this type of shell motions. Frequency responses of the nonlinear modes and traveling waves are qualitatively similar. This is explained by the similarity of systems of nonlinear algebraic equations with respect to vibrations amplitudes.

Nonlinear vibrations of cylindrical shell in supersonic flow at the outer part of the shell are treated in Refs. [32,33]. The Donnell equations (Eq. (28)) are used to describe the shell vibrations with geometrical nonlinearity. The first-order linear piston theory is used to approximate the aerodynamic pressure:

$$
P=\chi P_{\infty}\left[\frac{1}{a_{\infty}} w_{t}+M_{\infty} w_{x}\right]
$$

where $\chi$ is the polytrophic exponent; $P_{\infty}$ is the free stream static pressure, and $M_{\infty}$ is Mach number. Shell deflection takes the following form:

$$
\begin{aligned}
w= & q_{1} \sin r x \sin s y+q_{2} \sin r x \cos s y+q_{3} \sin 2 r x \sin s y \\
& +q_{4} \sin 2 r x \cos s y+f^{*}(x, t)
\end{aligned}
$$

Using the inextensibility condition of the middle surface in Eq. (31), the following expression for $f^{*}$ can be obtained:

$$
f^{*}(x, t)=\frac{n^{2}}{4 R}\left[\left(q_{1} \sin r x+q_{3} \sin 2 r x\right)^{2}+\left(q_{2} \sin r x+q_{4} \sin 2 r x\right)^{2}\right]
$$

Shell vibrations without initial imperfections are described by the four-degree-of-freedom nonlinear dynamical system:

$$
\begin{aligned}
& \ddot{q}_{1}+\omega_{1}^{2} q_{1}+\alpha \dot{q}_{1}-\beta q_{3}=\mathrm{Z}_{1}\left(q_{i}, \dot{q}_{i}, \ddot{q}_{i}\right) \\
& \ddot{q}_{2}+\omega_{1}^{2} q_{2}+\alpha \dot{q}_{2}-\beta q_{4}=\mathrm{Z}_{2}\left(q_{i}, \dot{q}_{i}, \ddot{q}_{i}\right) \\
& \ddot{q}_{3}+\omega_{2}^{2} q_{3}+\alpha \dot{q}_{3}+\beta q_{1}=\mathrm{Z}_{3}\left(q_{i}, \dot{q}_{i}, \ddot{q}_{i}\right) \\
& \ddot{q}_{4}+\omega_{2}^{2} q_{4}+\alpha \dot{q}_{4}+\beta q_{2}=\mathrm{Z}_{4}\left(q_{i}, \dot{q}_{i}, \ddot{q}_{i}\right)
\end{aligned}
$$

where $\alpha=\chi P_{\infty} / \rho h a_{\infty} ; \beta=(8 / 3)\left(\chi P_{\infty} M_{\infty} / L h \rho\right) ; Z_{i} ; i=1, \ldots, 4$ are nonlinear terms. The self-sustained vibrations of the shell are described by NNMs. The modal line projections are close to the rectilinear curve $q_{1}=q_{2}, q_{3}=q_{4}$ and this line is close to ellipse in plane $\left(q_{1}, q_{3}\right)$. Figure 5 shows the results of the NNMs numerical simulations in plane $\left(q_{1}, q_{3}\right)$.

Influence of the cylindrical shell initial imperfections on the self-sustained vibrations in supersonic flow is analyzed in the Ref. [33]. Initial imperfections and the dynamical deflection are chosen in the form of Eqs. (29) and (40), respectively. Analysis of the four-degree-of-freedom dynamical system shows that NNMs are observed in the flutter region. These NNMs are close to the rectilinear modal lines, which are observed in the system without the flow.

$q_{3}$

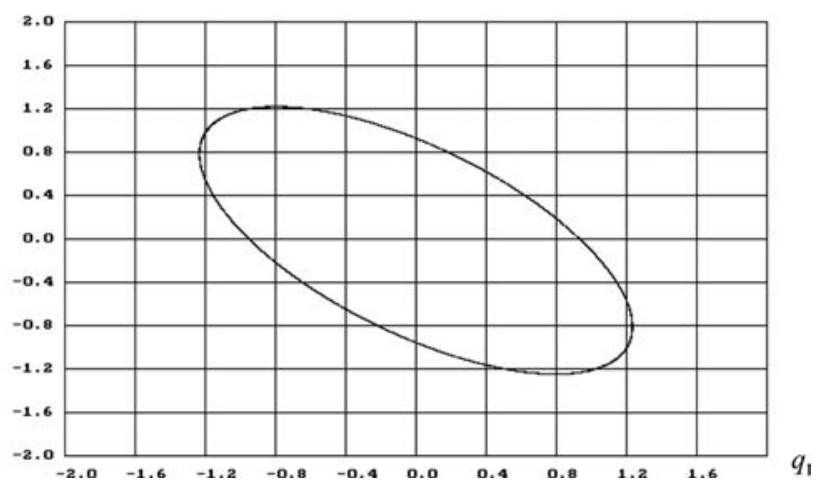

Fig. 5 NNMs of the self-sustained vibrations in plane $\left(P_{0}+P_{t} \cos 2 \Omega t\right)$ 


\section{Concept of Shaw-Pierre NNMs for Analysis of Mechanical System Dynamics and Its Application}

3.1 Fundamentals of General Theory. The KaudererRosenberg NNMs can be used to analyze large amplitudes motions. In particular, snap-through motions, when the system jumps between three equilibrium positions, are described by such NNMs. However, the Kauderer-Rosenberg NNMs are mostly used to analyze mechanical systems without damping. The Shaw-Pierre NNMs do not possess this shortcoming. Fundamentals of the Shaw-Pierre NNMs theory are treated in Ref. [34]. The Shaw-Pierre concept is based on a determination of invariant manifolds. Finite-degree-of-freedom mechanical system in Eq. (3) is considered. The damping can be taken into account. This system can be presented in a phase-space as:

$$
\begin{aligned}
& \dot{q}_{i}=y_{i} \\
& \dot{y}_{i}=f_{i}(q, y) \\
& i=1, \ldots, n
\end{aligned}
$$

where $y=\left[y_{1}, y_{2}, \ldots, y_{n}\right]^{T}$. Following the Shaw-Pierre concept, all phase variables of the system in Eq. (43) are defined by two "master" (active) coordinates $u \equiv q_{1} ; v \equiv y_{1}$. Other phase coordinates are named "slave" coordinates. NNM can be presented as

$$
\begin{aligned}
& q_{i}=Z_{i}(u, v) ; \quad y_{i}=Y_{i}(u, v) \\
& i=1, \ldots, n
\end{aligned}
$$

where $Z_{1}(u, v)=u ; Y_{1}(u, v)=v$. Functions $Z_{i}(u, v) ; Y_{i}(u, v)$ are determined from the following system of nonlinear partial differential equations:

$$
\begin{gathered}
Y_{i}(u, v)=\frac{\partial Z_{i}(u, v)}{\partial u} v+\frac{\partial Z_{i}(u, v)}{\partial v} f_{1}\left[u, Z_{2}(u, v), \ldots, Z_{n}(u, v) ;\right. \\
\left.v, Y_{2}(u, v), \ldots, Y_{n}(u, v)\right] \\
f_{i}\left[u, Z_{2}(u, v), \ldots, Z_{n}(u, v) ; v ; Y_{2}(u, v), \ldots, Y_{n}(u, v)\right] \\
=\frac{\partial Y_{i}(u, v)}{\partial u} v+\frac{\partial Y_{i}(u, v)}{\partial v} f_{1}\left[u, Z_{2}(u, v), \ldots, Z_{n}(u, v) ;\right. \\
\left.v ; Y_{2}(u, v), \ldots, Y_{n}(u, v)\right] \quad i=1, \ldots, n
\end{gathered}
$$

It is assumed, that the system of partial differential equations in Eq. (45) is solved and functions $Z_{2}(u, v), \ldots, Z_{n}(u, v)$; $Y_{2}(u, v), \ldots, Y_{n}(u, v)$ are determined. Obtained functions are substituted into the first two equations of the system in Eq. (43). As a result, the following one-degree-of-freedom dynamical system is derived:

$$
\dot{u}=v ; \quad \dot{v}=f_{1}\left[u, Z_{2}(u, v), \ldots, Z_{n}(u, v) ; v, Y_{2}(u, v), \ldots, Y_{n}(u, v)\right]
$$

The system in Eq. (46) dynamics can be analyzed by analytical or numerical methods. In particular, the harmonic balance method can be used.

Shaw and Pierre suggest several approaches to solve the system in Eq. (45). In particular, if vibrations close to the equilibrium position $q_{i}=y_{i}=0 ; i=1, \ldots, n$ are analyzed, power series are used to solve the system in Eq. (45), see Ref. [34]. NNMs take the form:

$$
\begin{aligned}
& Z_{i}(u, v)=a_{1 i} u+a_{2 i} v+a_{3 i} u^{2}+a_{4 i} u v+a_{5 i} v^{2}+\cdots \\
& Y_{i}(u, v)=b_{1 i} u+b_{2 i} v+b_{3 i} u^{2}+b_{4 i} u v+b_{5 i} v^{2}+\cdots \\
& i=2, \ldots, n
\end{aligned}
$$

where $a_{1 i} ; a_{2 i} ; \ldots ; b_{1 i} ; \ldots$ are unknown coefficients. In order to obtain NNM, the series in Eq. (47) are substituted into the system of partial differential equations in Eq. (45) and coefficients at the same powers $u^{i} v^{j} ; i=0,1, \ldots ; j=0,1, \ldots$ are equated. As a result, a system of algebraic equations with respect to coefficients of expansions in Eq. (47) is obtained [34].

NNM-based Galerkin projections are used to solve the system of partial differential equations in Eq. (45) in Refs. [35,36].

A method for construction of forced vibrations NNMs is used for the following two-degree-of-freedom system in Ref. [36]:

$$
\begin{aligned}
& m_{1} \ddot{q}_{1}+k_{1} q_{1}+\beta_{1} q_{1}^{3}+k_{2}\left(q_{1}-q_{2}\right)=f \cos \left(\omega_{f} t\right) \\
& m_{2} \ddot{q}_{2}+k_{2}\left(q_{2}-q_{1}\right)+k_{3} q_{2}+\beta_{2} q_{2}^{3}=0
\end{aligned}
$$

The system in Eq. (48) is transformed into principal coordinates $\left(\eta_{1}, \eta_{2}\right)$ and the linear damping is taken into account. Then the system in Eq. (48) has the following form:

$$
\begin{aligned}
& \ddot{\eta}_{i}+2 \xi_{i} \omega_{i} \dot{\eta}_{i}+\omega_{i}^{2} \eta_{i}=F_{i}\left(\eta_{1}, \eta_{2}\right)+f_{i} \cos \left(\omega_{f} t\right) \\
& i=1,2
\end{aligned}
$$

Master coordinates $\left(\eta_{1}, y_{1} \equiv \dot{\eta}_{1}\right)$ are transformed into polar coordinates $\eta_{1}=a \cos \phi ; \dot{\eta}_{1}=-\omega_{1} a \sin \phi$. NNM of the system in Eq. (49) takes the form

$$
\eta_{2}=P_{2}\left(a, \phi, \phi_{f}\right) ; \quad y_{2} \equiv \dot{\eta}_{2}=Q_{2}\left(a, \phi, \phi_{f}\right)
$$

where $\phi_{f}=\omega_{f} t$. Then motions on NNM are described by the following equations [36]:

$$
\begin{aligned}
& \dot{a}=-2 \xi_{1} a \omega_{1} \sin ^{2} \phi-\omega_{1}^{-1}\left(F_{1}+f_{1} \cos \phi_{f}\right) \sin \phi \\
& \dot{\phi}=\omega_{1}-\xi_{1} \omega_{1} \sin 2 \phi-\omega_{1}^{-1} a^{-1}\left(F_{1}+f_{1} \cos \phi_{f}\right) \cos \phi \\
& \dot{\phi}_{f}=\omega_{f}
\end{aligned}
$$

NNM in Eq. (50) is determined from the following nonlinear partial differential equations:

$$
\begin{aligned}
Q_{2}= & \frac{\partial P_{2}}{\partial a}\left[-2 \xi_{1} a \omega_{1} \sin ^{2} \phi-\omega_{1}^{-1}\left(F_{1}+f_{1} \cos \phi_{f}\right) \sin \phi\right]+ \\
& +\frac{\partial P_{2}}{\partial \phi}\left[\omega_{1}-\xi_{1} \omega_{1} \sin 2 \phi-\omega_{1}^{-1} a^{-1}\left(F_{1}+f_{1} \cos \phi_{f}\right) \cos \phi\right] \\
& +\frac{\partial P_{2}}{\partial \phi_{f}} \omega_{f}-2 \xi_{2} \omega_{2} Q_{2}-\omega_{2}^{2} P_{2}+F_{2}+f_{2} \cos \phi_{f} \\
= & \frac{\partial Q_{2}}{\partial a}\left[-2 \xi_{1} a \omega_{1} \sin ^{2} \phi-\omega_{k}^{-1}\left(F_{2}+f_{2} \cos \phi_{f}\right) \sin \phi\right] \\
& +\frac{\partial Q_{2}}{\partial \phi}\left[\omega_{1}-\xi_{1} \omega_{1} \sin 2 \phi-\omega_{1}^{-1} a^{-1}\left(F_{1}+f_{1} \cos \phi_{f}\right) \cos \phi\right] \\
& +\frac{\partial Q_{i}}{\partial \phi_{f}} \omega_{f}
\end{aligned}
$$

The method of solution of nonlinear partial differential equations in Eq. (52) is discussed in Ref. [36].

3.2 Analysis of the Finite-Degree-of-Freedom Mechanical System. The Shaw-Pierre NNMs are widely used in applied mechanics. In this subsection, the dynamics of a finite-degree-offreedom system is considered.

Coupled nonlinear dynamics of floating offshore platform is treated in Ref. [37]. System dynamics is described by six-degreeof-freedom nonlinear system. The Shaw-Pierre NNMs are used to investigate a damped system dynamics. In-plane and out-of-plane normal oscillation modes of a vessel at the critical orientation angle and around the zero in-plane tilt angle are treated. It can be inferred from the results that around the zero equilibrium vessel executes small amplitude in-plane oscillations. The NNMs analysis allows for the capture of the nonlinear interaction of the saddle 
type between these two modes, which could not be captured using a linearized approach. For this vessel, near the stable orbit at the zero in-plane tilt angle, the out-of-plane dynamics is of the saddle type and the out-of-plane restoring is relatively small. However, for the saddle orbit near the in-plane angle of vanishing stability, the out of plane restoring becomes dominate in a relative sense. Moreover, NNMs allow infer what will happen under external wave excitation. In-plane and out-of-plane resonances are expected which cannot be accurately predicted using a linearized and uncoupled analysis.

Vertical dynamics of a double-tracked road vehicle with sevendegrees-of-freedom is considered in Refs. [38,39]. The car is described by a rigid body. The heave, roll, and pitch motions are treated. Vertical displacements, the pitch angle, and the roll angle are described by generalized coordinates $z, \alpha$, and $\beta$, respectively. Vertical displacements of the $\boldsymbol{i}^{\boldsymbol{t h}}$ suspended mass $x_{i}$ are equivalent to the wheel. Tires are described by elastic elements with linear characteristics. Suspension is determined by nonlinear elastic characteristics. Elastic forces of the suspensions $f_{1}(x)$ and $f_{2}(x)$ are approximated by polynomials. Displacements of the rigid body mass center are described by vector $q=[z, \alpha, \beta]^{T}$ and displacements of the suspended masses are determined by vector $x=\left[x_{1}, x_{2}, x_{3}, x_{4}\right]$. Difference between the displacements of the body and the elements of the suspension is $U=\left[U_{1}, \ldots, U_{4}\right]$ $=H q-x$, where $H$ is a four dimension matrix. Suspension nonlinear characteristics are denoted by $C_{N L}=\left[f_{1}\left(U_{1}\right), f_{1}\left(U_{2}\right)\right.$, $\left.f_{2}\left(U_{3}\right), f_{2}\left(U_{4}\right)\right]^{T}$. The system of the ordinary differential equations in the matrix form, which describes nonlinear vibrations of the car, is the following:

$$
\begin{aligned}
& M_{C} \ddot{q}+H^{T} C_{N L}+H^{T} K V=0 \\
& M_{S} \ddot{x}-C_{N L}+C x-K V=0
\end{aligned}
$$

where $V=H \dot{q}-\dot{x}$ is the velocity vector; $M_{C}$ is the matrix of the car inertia; $M_{S}$ is the matrix of the suspension inertia; $C$ is the matrix of tire stiffness, and $K$ is the damping matrix. Shaw-Pierre NNMs are used to study the dynamics of the system in Eq. (53).

If nonlinear elastic characteristics of suspensions are taken into account, the quarter-car model has piecewise linear or piecewise nonlinear characteristics of shock absorbers $[38,39]$. The invariant manifold with master coordinates $\left(u=x_{1}, v=\dot{x}_{1}\right)$ and the motion along the first NNM is presented in Fig. 6.

Motions of the rotor- bearing system are studied using the Shaw-Pierre NNMs in Ref. [40]. One-disk rotor dynamics is described by a two-degree-of-freedom system with the cubic nonlinearity of elastic characteristic. Two generalized coordinates describe the rotor dynamics in two perpendicular directions. Vibrations are described by the following system of ordinary differential equations in the matrix form [40]:

$$
M \ddot{q}+(C+\Omega G) \dot{q}+K q+\Gamma q^{3}=0
$$

where $M$ is the symmetric mass matrix; $K$ is the symmetric stiffness matrix; $C$ is the symmetric damping matrix, and $G$ is the skew symmetric gyroscopic matrix. Term $\Gamma q^{3}$ represents a stiffness type of nonlinearity with diagonal matrix of coefficients $\Gamma$. Two Shaw-Pierre NNMs are calculated in the system in Eq. (54).

Nonlinear dynamics of the one-disk rotor (Fig. 7) is treated in Refs. [41,42]. The rotor consists of a rigid disk and an elastic shaft; the mass of the shaft is not taken into account. The shaft is supported by two short-length journal bearings. As a result of the disk vibrations, the journals $\boldsymbol{A}$ and $\boldsymbol{B}$ are moved. These bearings motions are described by generalized coordinates $\left(x_{1}(t), y_{1}(t)\right)$ and $\left(x_{2}(t), y_{2}(t)\right)$. Two journal bearings are identical. The rotor rotates with constant angular velocity $\Omega$ about the $z$ axis. The rigid disk motions are described by four-degrees-of-freedom. Generalized coordinates $(x, y)$ and $\left(\theta_{1}, \theta_{2}\right)$ describe motions of the disk gravity center and the disk rotation about axes $x, y$, respectively. The analytical solution of the Reynolds equation for short-length

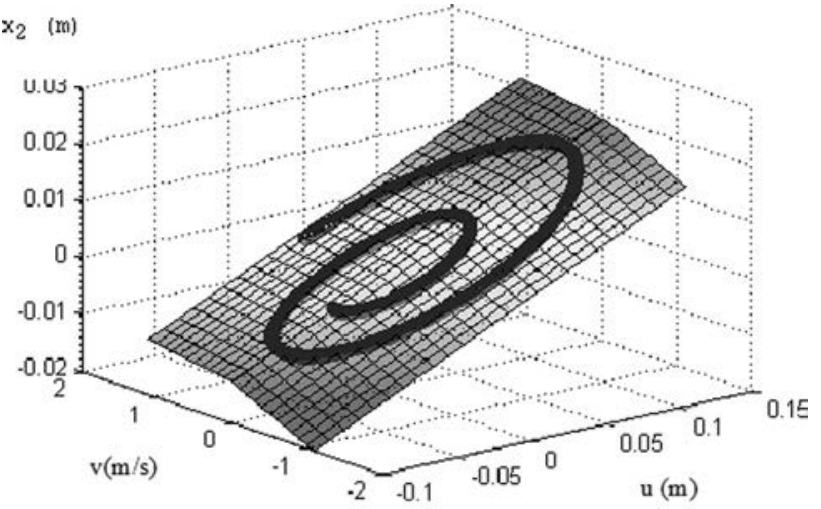

(a)

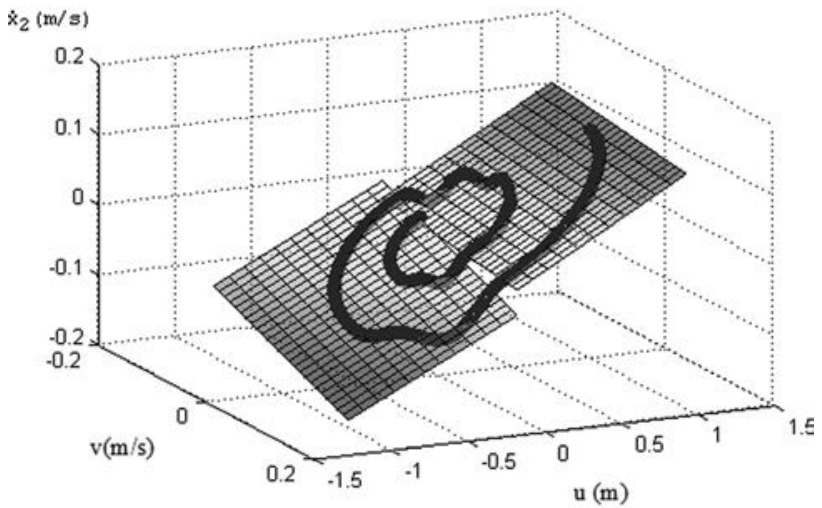

(b)

Fig. 6 NNM of a piecewise linear system with a shock absorber. Projections of invariant manifolds on $\left(u, v, x_{2}\right)$ and $(u$, $\left.v, x_{2}\right)$ are shown on figures $(a)$ and $(b)$, respectively.

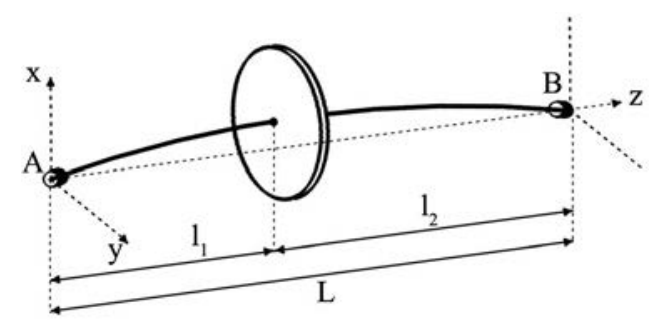

Fig. 7 Outline of the one-disk rotor

bearing is used to obtain the pressure acting on the journals. The rotor dynamics is described by the following equations:

$$
\begin{aligned}
& m \ddot{x}-\tilde{F}_{x}\left(x_{2}, y_{2}\right)-\tilde{F}_{x}\left(x_{1}, y_{1}\right)=0 \\
& I_{e} \ddot{\theta}_{2}-I_{p} \Omega \dot{\theta}_{1}-l_{2} \tilde{F}_{x}\left(x_{2}, y_{2}\right)+l_{1} \tilde{F}_{x}\left(x_{1}, y_{1}\right)=0 \\
& m \ddot{y}-\tilde{F}_{y}\left(x_{1}, y_{1}\right)-\tilde{F}_{y}\left(x_{2}, y_{2}\right)=0 \\
& I_{e} \ddot{\theta}_{1}+I_{p} \Omega \dot{\theta}_{2}-l_{1} \tilde{F}_{y}\left(x_{1}, y_{1}\right)+l_{2} \tilde{F}_{y}\left(x_{2}, y_{2}\right)=0
\end{aligned}
$$

where $m, I_{D}, I_{e}$ are mass and moments of the disk inertia; $\tilde{F}_{x}\left(x_{1}, y_{1}\right), F_{y}\left(x_{1}, y_{1}\right)$ and $\tilde{F}_{x}\left(x_{2}, y_{2}\right), \tilde{F}_{y}\left(x_{2}, y_{2}\right)$ are projections of forces acting on the bearings $A$ and $B$, respectively. Nonlinear forces $\tilde{F}_{x}, \tilde{F}_{y}$ are expanded into the Taylor series and dimensionless variables and parameters are used. As a result, the following dynamical system in matrix form is obtained [41,42]:

$$
[\mathrm{M}] \ddot{q}+[\mathrm{G}] \dot{q}=\left[\mathrm{K}_{1}\right] q+\left[\mathrm{D}_{1}\right] q^{\prime}+W\left(q_{1}, q_{1}^{\prime}\right)
$$


where $q=\left[x, \theta_{1}, y, \theta_{2}\right]^{T}, q_{1}=\left[\tilde{x}_{1}, \tilde{y}_{1}, \tilde{x}_{2}, \tilde{y}_{2}\right]^{T} ; q$ is a vector of the disk generalized coordinates; $q_{1}$ is a vector of the generalized coordinates of the bearings, and $W=\left(w_{1}, \ldots, w_{4}\right)^{T}$ is the nonlinear part of the fluid film forces. The Shaw-Pierre NNMs are used to analyze self-sustained vibrations of the system in Eq. (56) with small amplitudes. Unstable limit cycles originate due to the Hopf bifurcation. These limit cycles undergo saddle-node bifurcation. The origin of the rotor self-sustained vibrations is studied by the NNM approach. The harmonic balance method and shooting technique are used to analyze the rotor self-sustained vibrations with moderate amplitudes. As follows from the results of the numerical analysis of the rotor self-sustained vibrations, both monoharmonic and polyharmonic vibrations are observed.

Nonlinear dynamics of the one-disk rotor in two ball bearings (Fig. 7) is considered in Refs. [39,43-45]. The ball bearings on the nonlinear supports are placed in the points $A$ and $B$. These bearings are presented by the lumped masses. Nonlinear springs are attached to these masses to describe nonlinear elastic properties of the bases. The rotor motions are described by eight generalized coordinates. The gravity center of the disk is placed on the distance from the rotor rotation axis. Therefore, the forced vibrations of the one-disk unbalanced rotor with the elastic shaft on nonlinear flexural base are considered. Gyroscopic effects, nonlinear flexible base, inertial forces of supports and internal resonances are taken into account. Equations of the system forced vibrations are the following:

$$
\begin{aligned}
& m \ddot{x}+\rho_{1} \dot{x}+c_{11}\left(x-h_{1} x_{2}-h_{2} x_{1}\right)+c_{12}\left(\theta_{2}-\frac{x_{2}-x_{1}}{l}\right) \\
& \quad=\varepsilon \Omega^{2} m \cos \Omega t \\
& m \ddot{y}+\rho_{1} \dot{y}+c_{11}\left(y-h_{1} y_{2}-h_{2} y_{1}\right)+c_{12}\left(-\theta_{1}-\frac{y_{2}-y_{1}}{l}\right) \\
& \quad=\varepsilon \Omega^{2} m \sin \Omega t \\
& I_{e} \ddot{\theta}_{1}+\rho_{2} \dot{\theta}_{1}+I_{p} \Omega \dot{\theta}_{2}-c_{21}\left(y-h_{1} y_{2}-h_{2} y_{1}\right)+c_{22}\left(\theta_{1}+\frac{y_{2}-y_{1}}{l}\right) \\
& \quad=0 \\
& I_{e} \ddot{\theta}_{2}+\rho_{2} \dot{\theta}_{2}-I_{p} \Omega \dot{\theta}_{1}+c_{21}\left(x-h_{1} x_{2}-h_{2} x_{1}\right)+c_{22}\left(\theta_{2}-\frac{x_{2}-x_{1}}{l}\right) \\
& \quad=0 \\
& m_{1} \ddot{x}_{1}+\beta \dot{x}_{1}+s_{1}\left(x-h_{1} x_{2}-h_{2} x_{1}\right)+s_{2}\left(\theta_{2}-\frac{x_{2}-x_{1}}{l}\right) \\
& \quad+c_{x}^{(1)} x_{1}+c_{x}^{(2)} x_{1}^{3}=0 \\
& m_{1} \ddot{y}_{1}+\beta \dot{y}_{1}+s_{1}\left(y-h_{1} y_{2}-h_{2} y_{1}\right)-s_{2}\left(\theta_{1}+\frac{y_{2}-y_{1}}{l}\right) \\
& \quad+c_{y}^{(1)} y_{1}+c_{y}^{(2)} y_{1}^{3}=0 \\
& m_{2} \ddot{x}_{2}+\beta \dot{x}_{2}+s_{3}\left(x-h_{1} x_{2}-h_{2} x_{1}\right)+s_{4}\left(\theta_{2}-\frac{x_{2}-x_{1}}{l}\right) \\
& \quad+k_{x}^{(1)} x_{2}+k_{x}^{(2)} x_{2}^{3}=0 \\
& m_{2} \ddot{y}_{2}+\beta \dot{y}_{2}+s_{3}\left(y-h_{1} y_{2}-h_{2} y_{1}\right)-s_{4}\left(\theta_{1}+\frac{y_{2}-y_{1}}{l}\right) \\
& \quad+k_{y}^{(1)} y_{2}+k_{y}^{(2)} y_{2}^{3}=0
\end{aligned}
$$

where $\rho_{1}, \rho_{2}$ are parameters of the disk linear damping; $c_{11}, c_{12}$, $c_{22}$ are elements of the shaft stiffness matrix; $c_{x}^{(1)}, k_{x}^{(1)}, c_{y}^{(1)}, k_{y}^{(1)}$ are linear stiffness coefficients of the base, and $c_{x}^{(2)}, k_{x}^{(2)}, c_{y}^{(2)}, k_{y}^{(2)}$ are coefficients of nonlinear stiffness. Generalized coordinates and notations of the parameters of the system in Eq. (57) coincide with notations of the system in Eq. (55).

Masses of the rotor journals bearings are not taken into account in Ref. [43]. Then the last four equations of the system in Eq. (57) describe equilibrium of the journal bearings. Thus, equations of the system motions consist of four nonlinear ordinary differential equations and four nonlinear algebraic equations. The supports inertia is taken into account in Refs. [39,44,45].
The Shaw-Pierre NNMs in conjunction with the modified Rauscher method $[2,4,46]$ are used to analyze forced nonlinear vibrations of the system in Eq. (57) with 1:1 internal resonance. NNMs approach permits to reduce eight-degree-of-freedom nonlinear of the system in Eq. (57) to a two-degree-of-freedom nonlinear system. These reduced systems are analyzed by the harmonic balance method. The frequency response near the first resonance is calculated. The disk gravity center performs motions, which are essentially different from a circle. The trajectories of the resonance vibrations in the system configuration space are shown in Fig. 8. Stability analysis of the NNMs shows that, in this frequency range, a pair of new motions is appeared. These motions can be found by the NNMs approach too. In these regimes, the disk center circumscribes elliptic-type trajectories.

Warminski [47] analyzed the self-sustained vibrations of twodegree-of-freedom mechanical system:

$$
\begin{aligned}
& m_{1} \ddot{q}_{1}+\left(\beta_{1} \dot{q}_{1}^{2}-\alpha_{1}\right) \dot{q}_{1}+\delta_{1} q_{1}+\gamma_{1} q_{1}^{3}+\delta_{12}\left(q_{1}-q_{2}\right)=0 \\
& m_{2} \ddot{q}_{2}+\left(\beta_{2} \dot{q}_{2}^{2}-\alpha_{2}\right) \dot{q}_{2}+\delta_{2} q_{2}+\gamma_{2} q_{2}^{3}-\delta_{12}\left(q_{1}-q_{2}\right)=0
\end{aligned}
$$

The Shaw-Pierre NNMs are determined. NNMs are applied to decouple motions of the system. Two separate limit cycles have been obtained. For a system with four potential wells the response can be regular or chaotic.

Free vibrations of piecewise linear $n$-degree-of-freedom system are treated by Chen and Shaw [48]. This system takes the following form:

$$
\begin{cases}M \ddot{q}+K_{1} q=0 ; & h^{T} q \leq d^{\prime} \\ M \ddot{q}+K_{2} q=b ; & h^{T} q>d^{\prime}\end{cases}
$$

where $h, b \in R^{n} ; M, K_{1}, K_{2}$ are real, symmetric, positively definite $n \times n$ matrix. Analytical solutions for two linear parts are used to construct the Poincare sections. It is assumed that the first generalized velocity on the Poincare sections $h^{T} q=d^{\prime}$ is small. The asymptotic solutions for the Poincare sections are constructed by this generalized velocity. It is justified that the obtained motions are NNMs. From the expression for these NNMs, the modal dynamics and the frequency responses are obtained.

Piecewise linear $n$-degree-of-freedom system is treated in Ref. [49]. This system can be presented in the following form:

$$
\ddot{\eta}+\Lambda_{1} \eta=f(\eta)
$$

where $\eta \in R^{n}$ is the vector of modal co-ordinates; $\Lambda_{1}$ is $n \times n$ diagonal matrix; $f(\eta) \in R^{n}$ is piecewise linear vector of forces. The Galerkin approach is applied to construct NNMs for the piecewise linear systems. Transformation from master coordinates $\left(\eta_{k}, \dot{\eta}_{k}\right)$ to amplitude and phase co-ordinates $(a, \phi)$ is the following:

$$
\begin{aligned}
& \eta_{k}(t)=a(t) \cos \phi(t) \\
& \dot{\eta}_{k}(t)=-\omega_{k} a(t) \sin \phi(t)
\end{aligned}
$$

where $\omega_{k}$ is the $\boldsymbol{k}^{\text {th }}$ natural frequency of the linear system in the first region of Eq. (59). On the invariant manifold, all slave coordinates are functions of master coordinates $(a, \phi)$ :

$$
\begin{aligned}
& \eta_{i}=P_{i}(a, \phi) \\
& \dot{\eta}_{i}=Q_{i}(a, \phi) ; \quad i=1, \ldots, n ; i \neq k
\end{aligned}
$$

Functions $P_{i}, Q_{i}$ satisfy the following system of partial differential equations: 

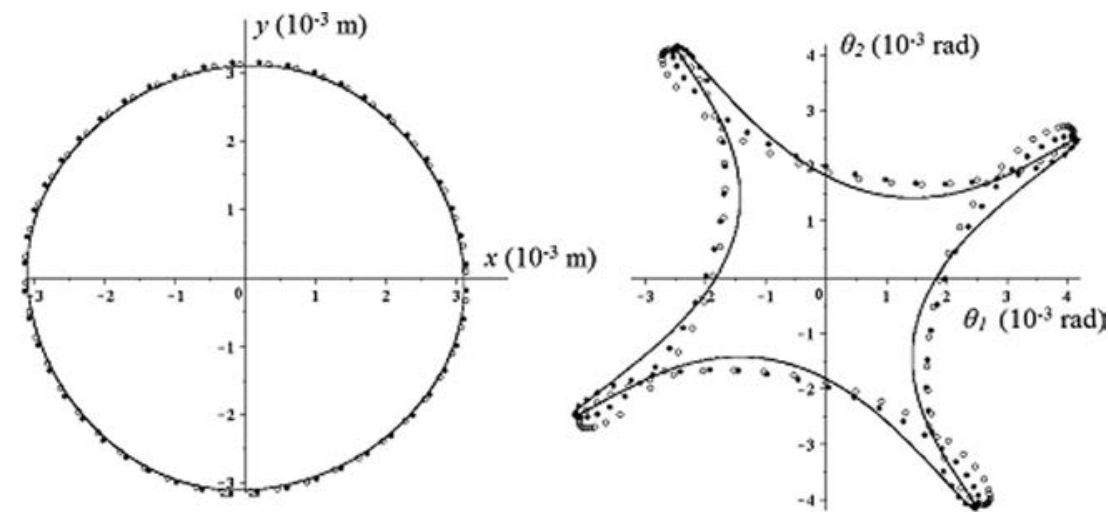

Fig. 8 Trajectories of the resonance motions in configuration space. Points and circles denote results, which are obtained by harmonic balance method and NNM approaches, respectively. The results of numerical simulations are shown by solid lines.

$$
\begin{aligned}
Q_{i} & =-\frac{f_{k}}{\omega_{k}} \frac{\partial P_{i}}{\partial a} \sin \phi+\frac{\partial P_{i}}{\partial \varphi}\left(\omega_{k}-\frac{f_{k} \cos \phi}{a \omega_{k}}\right) \\
-\omega_{i}^{2} P_{i}+f_{i} & =-\frac{f_{k}}{\omega_{k}} \frac{\partial Q_{i}}{\partial a} \sin \phi+\frac{\partial Q_{i}}{\partial \phi}\left(\omega_{k}-\frac{f_{k} \cos \phi}{a \omega_{k}}\right) \\
i & =1, \ldots, n ; i \neq k
\end{aligned}
$$

The Galerkin method is used to analyze the system in Eq. (63). The solution of this system is presented in the form of truncated series:

$$
\begin{aligned}
P_{i}(a, \phi) & =\sum_{l=1} \sum_{m=1} C_{i, l, m} T_{l, m}(a, \phi) \\
Q_{i}(a, \phi) & =\sum_{l=1} \sum_{m=1} D_{i, l, m} U_{l, m}(a, \phi)
\end{aligned}
$$

where $T_{l, m} ; U_{l, m}$ are trial functions and $C_{i, l, m} ; D_{i, l, m}$ are unknown coefficients.

The series in Eq. (64) is substituted into Eq. (63) and weak solutions are constructed. A system of nonlinear algebraic equations with respect to $C_{i l m}$ and $D_{i, l, m}$ is derived. Solving this system of nonlinear algebraic equations, the NNM Eq. (64) is obtained.

The dynamic response on each NNM manifold can be reduced to a single-degree-of-freedom system described in terms of master coordinates. Dynamic behavior of the piecewise linear systems NNM can be quite complicated. This includes loops in manifolds and instabilities leading to a variety of system responses. Stability and postcritical dynamics of NNMs are investigated using characteristic multipliers and Poincare map. Flip bifurcations and transitions to quasi-periodic response are found.

\subsection{Analysis of Discretized Structure Systems Using the} Shaw-Pierre NNMs

3.3.1 Beams Structures. Applications of the Shaw-Pierre NNMs for bending vibrations of beams, which are supported by nonlinear springs, are treated in Refs. [35,50,51]. Beam bending vibrations are described by the following partial differential equation [50]:

$$
\rho A \ddot{w}+E I w_{x x x x}+\gamma w^{3} \delta(x-0.5)=0
$$

where $\rho$ is a density of the beam material; $A, E, I$ are typical beam parameters, and $\delta(x-0.5)$ is the $\delta$-function. Beam bending vibrations are expanded using their eigenmodes. The obtained $\boldsymbol{n}$-degree-of-freedom dynamical system is studied using the Shaw-Pierre NNMs.

The finite element code PATRAN is used to obtain a linear model of beam transverse vibrations using 200 two-noded beam finite elements [35,51]. The beam is pinned at one end and the other one is constrained by a linear spring. A nonlinear torsional spring with quadratic and cubic nonlinearities is located at the pinned end. The Galerkin-based approach is used to analyze NNMs. The finite-element based calculations emphasizes that practical application of NNMs is not limited to highly idealized, analytically tractable systems.

Longitudinal-flexural vibrations of the Euler-Bernoulli rotating cantilever beam (Fig. 9), which is the model of rotorcraft blade, are treated in Refs. [52,53]. Beam geometrical nonlinearity is taken into account. Longitudinal displacements of the beam are divided into static equilibrium $u_{s}(x)$ and vibrations $u_{d}(x, t)$. Vibrations of the beam are described by the following system of the partial differential equations:

$$
\begin{aligned}
& u_{s, x x}+\lambda^{2} u=-\lambda^{2}(h+x) \\
& m \ddot{u}_{d}-m \Omega^{2} u_{d}-E A u_{d, x x}=0 \\
& m \ddot{w}+E I w_{x x x x}-E A\left(u_{s, x} w_{x x}+u_{s, x x} w_{x}\right)=0
\end{aligned}
$$

Static displacements are obtained exactly in the analytical form. Motions $u_{d}, w$ are expanded into truncated series using the trial functions. Using the Galerkin method, the finite-degree-of-freedom dynamical system is obtained. Then, the nonlinear equations of motion are reduced using a procedure based on the invariant manifold formulation of NNMs. The invariant manifold solution is shown to be quite accurate and the corresponding single degree of freedom reduced-order model yields results which agree nearly exactly with those of the original system. This approach accurately models the critical dynamic coupling between the axial and transverse beam displacements, as well as the coupling within the set of transverse modes through amplitudes of considerable magnitude.

Nonlinear vibrations of rotating beam (Fig. 9), which are described by the system in Eq. (66), are analyzed in Ref. [54]. Internal resonance is taken into account. Multimode invariant manifolds are used to analyze the nonlinear vibrations.

Vibrations of a clamped-clamped beam are analyzed taking into account nonlinear relations for longitudinal force in Ref. [55]. This system is described by the following integro-partial-differential equation:

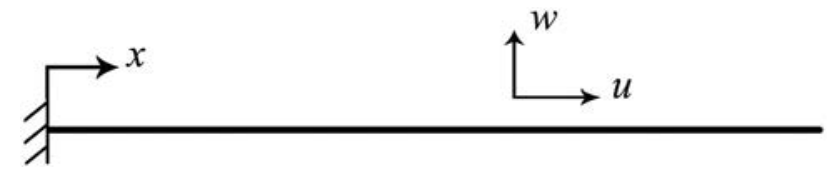

Fig. 9 Outline of rotating beams. Adapted from Ref. [52]. 


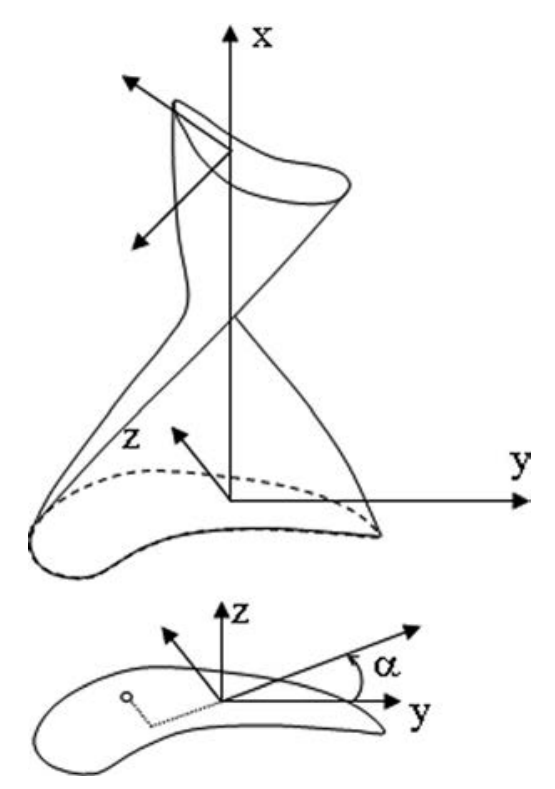

Fig. 10 Pretwisted beam with variable cross section

$$
m \ddot{w}+E I w_{x x x x}-\frac{E A}{2 L} w_{x x} \int_{o}^{L} w_{x}^{2} d x=0
$$

Notations of the system in Eq. (65) are retained. Using the standard Galerkin approach, the finite-degree-of-freedom dynamical system is derived. The Shaw-Pierre NNMs are applied to this dynamical system.

Flexural-flexural-torsional vibrations of a pretwisted beam with variable cross section (Fig. 10) are analyzed in Refs. [56,57]. The beam rotates with constant angular velocity $\Omega$. Geometrical nonlinearity is taken into account in the system model. A detailed derivation of the equations of such beam motions is presented in Ref. [56]. These equations have the following form:

$$
\begin{aligned}
& E\left(w^{\prime \prime} J_{\varsigma \eta}\right)^{\prime \prime}+E\left(v^{\prime \prime} J_{\varsigma}\right)^{\prime \prime}-m \varsigma^{(1)} \ddot{\theta}_{x}+m \ddot{v}+m \Omega^{2}\left[x v^{\prime}-x\left(\theta_{x} \varsigma^{(1)}\right)^{\prime}\right] \\
&+ L_{1}(u, v, w)=0 \\
& E\left(w^{\prime \prime} J_{\eta}\right)^{\prime \prime}+E\left(v^{\prime \prime} J_{\varsigma \eta}\right)^{\prime \prime}+m \eta^{(1)} \ddot{\theta}_{x}+m \ddot{w}+m \Omega^{2}\left[x w^{\prime}+x\left(\theta_{x} \eta^{(1)}\right)^{\prime}\right] \\
&+ L_{2}(u, v, w)=0 \\
&-\left(D_{\xi} \theta_{x}^{\prime}\right)^{\prime}+\left[m\left(\varsigma^{(1) 2}+\eta^{(1) 2}\right)+I_{\xi}\right] \ddot{\theta}_{x}+m \eta^{(1)} \ddot{w}-m \varsigma^{(1)} \ddot{v} \\
&+ \Omega^{2}\left[m \varsigma^{(1)} v+I_{\varsigma \eta}(x)+x w^{\prime} m \eta^{(1)}-x v^{\prime} m \varsigma^{(1)}\right]+L_{3}(u, v, w)=0 \\
& L_{1}= 2 m \Omega^{2} \dot{v} v^{\prime}+\left(\theta_{x} \varsigma^{(1)}-v\right)^{\prime \prime} \int_{0}^{L}\left(2 \Omega m \dot{v}+\Omega^{2} m x\right) d x \\
&-2 E\left(\theta_{x} v^{\prime \prime} J_{\varsigma \eta}\right)^{\prime \prime}+E\left[\theta_{x} w^{\prime \prime}\left(J_{\varsigma}-J_{\eta}\right)\right]^{\prime \prime}-4 m \Omega \dot{v}\left(\theta_{x} \varsigma^{(1)}\right)^{\prime} \\
&-2 \Omega(m \dot{v})^{\prime} \theta_{x} \varsigma^{(1)} \\
& L_{2}= 2 \Omega m \dot{v} w^{\prime}-\left(w+\theta_{x} \eta^{(1)}\right)^{\prime \prime} \int_{x}^{L}\left(2 \Omega m \dot{v}+\Omega^{2} m x\right) d x \\
&+E\left[\theta_{x} v^{\prime \prime}\left(J_{\varsigma}-J_{\eta}\right)\right]^{\prime \prime}+2 E\left(\theta_{x} w^{\prime \prime} J_{\varsigma \eta}\right)^{\prime \prime}+4 m \Omega \dot{v}\left(\theta_{x} \eta^{(1)}\right)^{\prime} \\
&+2 \Omega \eta^{(1)} \theta_{x}(m \dot{v})^{\prime} \\
& L_{3}=\left(\varsigma^{(1)} v^{\prime \prime}-\eta^{(1)} w^{\prime \prime}\right) \int_{x}^{L}\left(2 \Omega m \dot{v}+\Omega^{2} m x\right) d x \\
&+E\left(w^{\prime \prime} J_{\varsigma \eta}+v^{\prime \prime} J_{\varsigma}\right) w^{\prime \prime}-E\left(w^{\prime \prime} J_{\eta}+v^{\prime \prime} J_{\varsigma \eta}\right) v^{\prime \prime}
\end{aligned}
$$

where $J_{\varsigma}, J_{\eta}, J_{\varsigma \eta}, D_{\xi}$ are inertia moments of the beam crosssection; $\left(\eta^{(1)}, \varsigma^{(1)}\right)$ are coordinates of the cross-section gravity

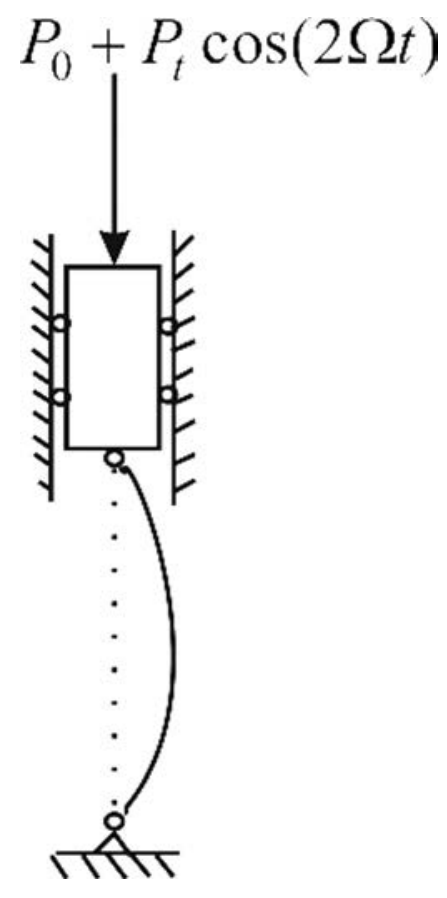

Fig. 11 Parametrically excited beam

center. The nonlinear system of the integro-partial-differential equation in Eq. (68) is transformed into a system of ordinary differential equations using the Galerkin approach. The Shaw-Pierre NNMs are used to reduce this system to one-degree-of-freedom oscillators, which describe motions on invariant manifolds. Influence of the cross-section warping on the backbone curve behavior is treated in Ref. [57]. It is shown that certain hard backbone curves, which are obtained without taking into account warping, are transformed into soft ones if warping is included.

Parametric vibrations of a flexible beam with a lumped mass at the end (Fig. 11) are treated in Ref. [58]. Vibrations of this system are described by the following integro-partial-differential equation:

$$
\begin{aligned}
& E J w_{x x x x}+0.5 E J\left(w_{x x} w_{x}^{2}\right)_{x x}+w_{x x}\left\{P_{t} \cos (2 \Omega t)-0.5 M \int_{0}^{L}\left(w_{x}^{2}\right)_{t t} d s\right\} \\
& \quad+m w_{t t}-\left(N w_{x}\right)_{x}=0 \\
& N=0.5 m \int_{s}^{l} d s_{1} \int_{0}^{s_{1}}\left(w_{x}^{2}\right)_{t t} d s_{2}
\end{aligned}
$$

where $P_{t} \cos (\Omega t)$ is the longitudinal force; $M$ is the mass attached to the beam. The summand $0.5 E J\left(w_{x x} w_{x}^{2}\right)_{x x}$ defines contribution of nonlinearity into the beam curvature; summands $0.5 M w_{x x}$ $\int_{0}^{L}\left(w_{x}^{2}\right)_{t t} d s,\left(N w_{x}\right)_{x}$ describe a nonlinear inertia. Transversal displacements are expanded using the first two eigenmodes of the beam. Beam vibrations are described by the following twodegree-of-freedom system:

$$
\begin{aligned}
\ddot{q}_{1} & +\pi^{4} q_{1}+\gamma_{1} q_{1}^{3}+\gamma_{2} q_{1} q_{2}^{2}+q_{1}\left[2 \gamma_{3}\left(\dot{q}_{1}^{2}+q_{1} \ddot{q}_{1}\right)+2 \gamma_{4}\left(\dot{q}_{2}^{2}+q_{2} \ddot{q}_{2}\right)\right. \\
& \left.+\gamma_{5}\left(q_{1} \ddot{q}_{2}+q_{2} \ddot{q}_{1}+2 \dot{q}_{1} \dot{q}_{2}\right)\right]+q_{2}\left[2 \gamma_{7}\left(\dot{q}_{1}^{2}+q_{1} \ddot{q}_{1}\right)\right. \\
& \left.+2 \gamma_{8}\left(\dot{q}_{2}^{2}+q_{2} \ddot{q}_{2}\right)+\gamma_{6}\left(q_{1} \ddot{q}_{2}+q_{2} \ddot{q}_{1}+2 \dot{q}_{1} \dot{q}_{2}\right)\right] \\
& -q_{1} f_{1} \cos (2 \Omega \tau)=0 \\
\ddot{q}_{2} & +16 \pi^{4} q_{2}+\beta_{1} q_{2}^{3}+q_{2}\left[2 \beta_{3}\left(\dot{q}_{1}^{2}+q_{1} \ddot{q}_{1}\right)+2 \beta_{4}\left(\dot{q}_{2}^{2}+q_{2} \ddot{q}_{2}\right)\right. \\
& \left.+\beta_{5}\left(q_{1} \ddot{q}_{2}+q_{2} \ddot{q}_{1}+2 \dot{q}_{1} \dot{q}_{2}\right)\right]+q_{1}\left[2 \beta_{7}\left(\dot{q}_{1}^{2}+q_{1} \ddot{q}_{1}\right)\right. \\
& \left.+2 \beta_{8}\left(\dot{q}_{2}^{2}+q_{2} \ddot{q}_{2}\right)+\beta_{6}\left(q_{1} \ddot{q}_{2}+q_{2} \ddot{q}_{1}+2 \dot{q}_{1} \dot{q}_{2}\right)\right] \\
& +\beta_{2} q_{2} q_{1}^{2}-4 q_{2} f_{1} \cos (2 \Omega \tau)=0
\end{aligned}
$$


A combination of the Rauscher method and the Shaw-Pierre NNMs are used to analyze the system in Eq. (70). Properties of parametric vibrations are treated using the frequency response. If the frequency of the parametric force is increased, the stable beam equilibrium is transformed into unstable one and unstable periodic motions originate. If the frequency of the parametric force is increased further, the unstable beam equilibrium becomes stable and stable periodic vibrations restore.

Bending vibrations of a simply supported beam on a nonlinear elastic base are analyzed in Ref. [59]. Beam vibrations are described by the following partial differential equation in dimensionless form:

$$
w_{t t}+w_{x x x x}+\alpha_{2} w^{2}+\alpha_{3} w^{3}=0
$$

The system in Eq. (71) is reduced to the finite-degree-of-freedom dynamical system, which is studied by nonlinear forms of ordinary differential equations.

Two-link flexible arms connected by a nonlinear torsion spring are considered in Ref. [60]. A three degree-of-freedom dynamical model describes the system vibrations. The multiple scales method is applied to construct NNMs.

Forced vibrations of a shallow arch, which are described by Eq. (17), are studied in Ref. [46]. In this case, the external timeperiodic excitation is included into Eq. (17). The dynamic deflection takes the following form:

$$
w(x, t)=\sum_{i=1}^{2}\left[\lambda_{i}+q_{i}(t)\right] \sin (i \pi x)
$$

where $\lambda_{i}$ describe small initial imperfections. A two-degree-offreedom nonlinear dynamical system is obtained in the form:

$$
\begin{aligned}
& \ddot{q}_{1}+\pi^{4} q_{1}+0.25 \pi^{4}\left(q_{1}+\lambda_{1}\right) \mu\left(q_{1}, q_{2}\right)=2 f \cos (\Omega t) \\
& \ddot{q}_{2}+16 \pi^{4} q_{2}+\pi^{4}\left(q_{2}+\lambda_{2}\right) \mu\left(q_{1}, q_{2}\right)=0 \\
& \mu\left(q_{1}, q_{2}\right)=q_{1}\left(q_{1}+2 \lambda_{1}\right)+4 q_{2}\left(q_{2}+2 \lambda_{2}\right)
\end{aligned}
$$

A combination of the Rauscher method and the Shaw-Pierre NNMs is used to analyze the system in Eq. (72). Frequency responses of the system forced vibrations are obtained. These responses in the regions of the main resonance have soft/hard behavior. The Shaw-Pierre NNMs are applied to analyze dynamics of frames in [61].

Nonlinear dynamics of rotating shaft supported by two journal bearings is investigated in Ref. [62]. The shaft flexural vibrations are expanded using the eigenmodes. Interaction of the journal bearings oil films with the shaft leads to self-sustained vibrations, which appear due the Hopf bifurcation. The Shaw-Pierre NNMs are used to analyze the shaft motions.

In Ref. [63], NNMs are used to analyze nonlinear vibrations of beams. The finite element method is used to obtain a finitedegree-of-freedom model of nonlinear vibrations. As a result, a thirty-degree-of-freedom mechanical system is obtained, which is analyzed by NNMs.

3.3.2 Plates and Shells Analysis. Self-sustained vibrations of a plate in a fluid flow are investigated in Ref. [64]. These vibrations are described by a high dimensional system of ordinary differential equations. As a result of the Hopf bifurcation, selfsustained vibrations of a plate arise. These motions are studied by the Shaw-Pierre NNMs.

A clamped shallow shell with complex base (Fig. 12) is investigated in Refs. [65,66]. The shell is thin; frequencies of longitudinal vibrations are significantly higher then frequencies of transverse motions. Therefore, longitudinal inertia and shear are not taken into account. Shells with geometrical nonlinearity are considered; then strains are small and displacements are moderate.

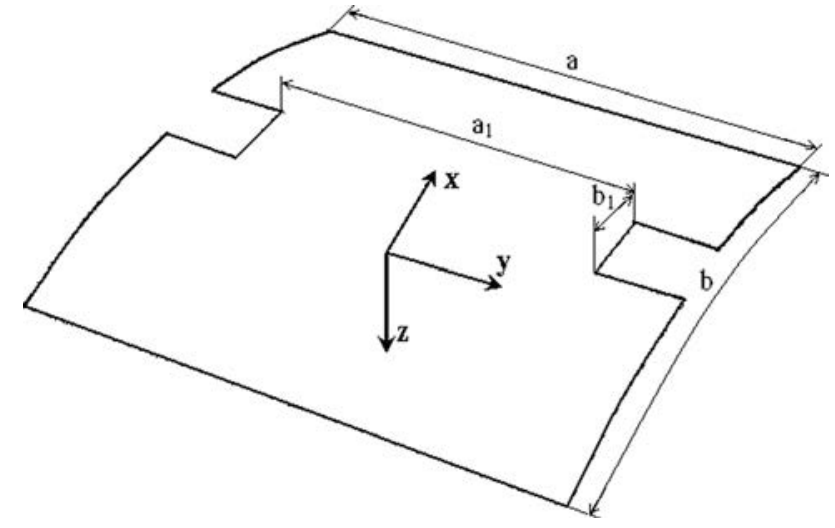

Fig. 12 Shallow shell with complex base

The Donnell equations with respect to $u, v, w$ are used to describe the shell dynamics:

$$
\begin{aligned}
& \frac{\partial^{2} u}{\partial x^{2}}+\frac{1-\mu}{2} \frac{\partial^{2} u}{\partial y^{2}}+\frac{1+\mu}{2} \frac{\partial^{2} v}{\partial x \partial y}-\left(K_{x}+\mu K_{y}\right) \frac{\partial w}{\partial x}+\frac{\partial w}{\partial x} \frac{\partial^{2} w}{\partial x^{2}} \\
& +\frac{1+\mu}{2} \frac{\partial w}{\partial y} \frac{\partial^{2} w}{\partial x \partial y}+\frac{1-\mu}{2} \frac{\partial w}{\partial x} \frac{\partial^{2} w}{\partial y^{2}}-\rho \frac{1-\mu^{2}}{E} \frac{\partial^{2} u}{\partial t^{2}}=0 \\
& \frac{\partial^{2} v}{\partial y^{2}}+\frac{1-\mu}{2} \frac{\partial^{2} v}{\partial x^{2}}+\frac{1+\mu}{2} \frac{\partial^{2} u}{\partial x \partial y}-\left(K_{y}+\mu K_{x}\right) \frac{\partial w}{\partial y}+\frac{\partial w}{\partial y} \frac{\partial^{2} w}{\partial y^{2}} \\
& +\frac{1+\mu}{2} \frac{\partial w}{\partial x} \frac{\partial^{2} w}{\partial x \partial y}+\frac{1-\mu}{2} \frac{\partial w}{\partial y} \frac{\partial^{2} w}{\partial x^{2}}-\rho \frac{1-\mu^{2}}{E} \frac{\partial^{2} v}{\partial t^{2}}=0 \\
& \frac{h^{2}}{12} \nabla^{4} w-\left(K_{x}+\mu K_{y}\right) \frac{\partial u}{\partial x}-\left(K_{y}+\mu K_{x}\right) \frac{\partial v}{\partial y} \\
& +\left(K_{x}^{2}+K_{y}^{2}+2 \mu K_{x} K_{y}\right) w-\frac{\left(K_{x}+\mu K_{y}\right)}{2}\left(\frac{\partial w}{\partial x}\right)^{2} \\
& \quad-\frac{\left(K_{y}+\mu K_{x}\right)}{2}\left(\frac{\partial w}{\partial y}\right)^{2}-\frac{\partial}{\partial x}\left(\frac{\partial w}{\partial x}\left(\frac{\partial u}{\partial x}+\mu \frac{\partial v}{\partial y}-\left(K_{x}+\mu K_{y}\right) w\right)\right. \\
& \left.+\frac{1-\mu}{2} \frac{\partial w}{\partial y}\left(\frac{\partial u}{\partial y}+\frac{\partial v}{\partial x}\right)\right)-\frac{\partial}{\partial y}\left(\frac { \partial w } { \partial y } \left(\mu \frac{\partial u}{\partial x}+\frac{\partial v}{\partial y}\right.\right. \\
& \left.\left.\quad+\left(K_{y}+\mu K_{x}\right) w\right)+\frac{1-\mu}{2} \frac{\partial w}{\partial x}\left(\frac{\partial u}{\partial y}+\frac{\partial v}{\partial x}\right)\right) \\
& +\rho \frac{1-\mu^{2}}{E} \frac{\partial^{2} w}{\partial t^{2}}=0(x, y) \in \Lambda
\end{aligned}
$$

where $K_{x}$ and $K_{y}$ are principal curvatures; $h$ is the shell thickness; $\rho$ is the material density, and $\Lambda$ is the shell base on the plane $(x, y)$. Displacements of the middle surface points are indicated by $u, v$, and $w$ in the $x, y$, and $z$ directions, respectively.

The R-function theory developed by Rvachov is used [7,67]. R-functions describe complex boundary of shallow shells bases. R-functions $\tilde{\omega}(x, y)$ are equal to zero on the shell boundary and positive inside the shell base $[65,68]$. Shell linear vibrations are presented as

$$
\begin{aligned}
& u(x, y, t)=\tilde{\omega}(x, y) P_{1}(x, y) \cos (\omega t) \\
& v(x, y, t)=\tilde{\omega}(x, y) P_{2}(x, y) \cos (\omega t) \\
& w(x, y, t)=\tilde{\omega}^{2}(x, y) P_{3}(x, y) \cos (\omega t)
\end{aligned}
$$

Nonlinear vibrations of the shell are expanded using eigenmodes, which contain R-functions. Applying the Galerkin method, a three-degree-of-freedom dynamical system with internal resonances is derived in the form of Eq. (43). A multimode invariant 


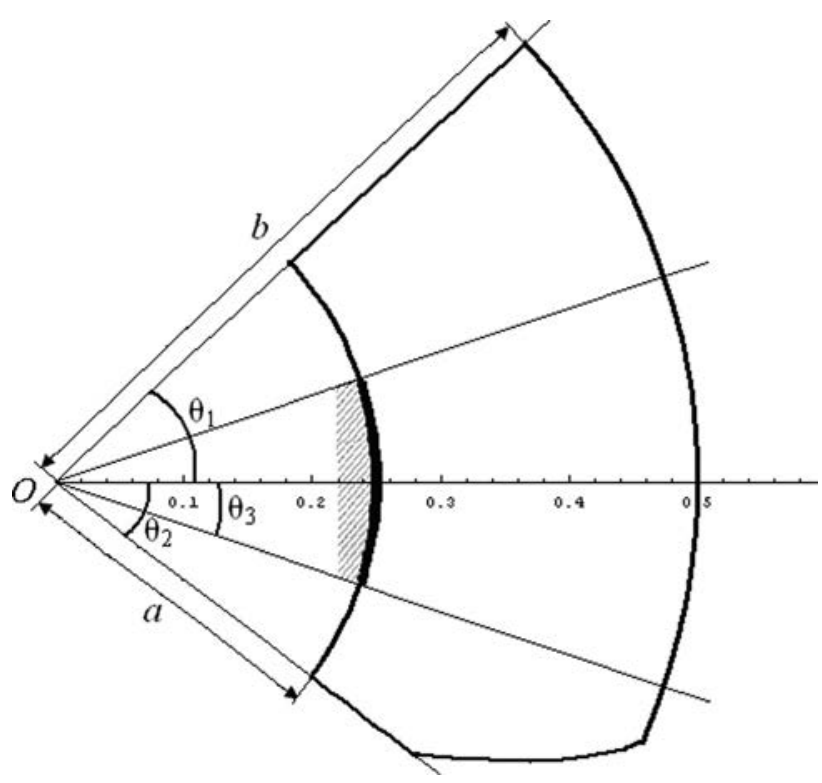

Fig. 13 Base of a shallow shell with variable thickness

manifold is used to analyze free nonlinear vibrations of the shell. Results of the analysis are shown on the frequency responses. Calculations of the plate free vibrations by one mode model are carried out. In this case, the backbone curve is soft. A three modes model of the plate is analyzed numerically. It is shown that behavior of a system with three degrees of freedom essentially differs from the free vibrations of a system with one degree of freedom. Stability of free nonlinear vibrations is lost at certain values of amplitudes and vibrations are unstable at greater values of amplitudes.

Vibrations of a shallow shell with a complex base and variable thickness are treated in Ref. [69]. The outline of the shell base is shown in Fig. 13. Shell vibrations in immovable, inviscid, incompressible, and potential fluid are analyzed. Potential energy of this shell takes the following form:

$$
\begin{aligned}
\Pi= & \frac{E}{2(1+\mu)} \int_{\Lambda}\left\{\frac { 1 } { 1 - \mu } \left[\left(\varepsilon_{11}^{2}+2 \mu \varepsilon_{11} \varepsilon_{22}+\varepsilon_{22}^{2}\right) h(\alpha, \beta)+\frac{h^{3}(\alpha, \beta)}{12}\right.\right. \\
& \left.\left.\times\left(\chi_{1}^{2}+2 \mu \chi_{1} \chi_{2}+\chi_{2}^{2}\right)\right]+\frac{1}{2}\left(\varepsilon_{12}^{2} h(\alpha, \beta)+\frac{1}{3} \kappa^{2} h^{3}(\alpha, \beta)\right)\right\} A B d \alpha d \beta
\end{aligned}
$$

where $\varepsilon_{11}, \varepsilon_{22}, \varepsilon_{12}$ are middle surface strains; $\chi_{1}, \chi_{2}, \kappa$ are curvatures and torsion of the middle surface; $h(\alpha, \beta)$ is the variable thickness of the shell; $A=\sqrt{g_{11}}$ and $B=\sqrt{g_{22}}$ are Lame parameters, and $g_{11}$ and $g_{22}$ are coefficients of the first quadratic form of the middle surface. The integrals in Eq. (77) are taken over the area of the shell base $\Lambda$ (Fig. 13). Two orthogonal directions, which are lines of the principle curvatures, are taken on the shell middle surface. Curvilinear coordinates $\alpha$ and $\beta$ are counted off along these lines. The third axis $z$ is directed orthogonally to the plane base.

Fluid motions around the shell are assumed to be nonrotational. The velocity potential $\Phi$ satisfies the Laplace equation. Boundary conditions on the shell surfaces have the following form: $\left.\Phi_{n}(x, t)\right|_{S^{ \pm}}=\left.w_{t}(x, t)\right|_{S^{ \pm}}$, where $\Phi(x, y)$ is the velocity potential; $S^{+}$ and $S^{-}$are upper and lower plate surfaces, respectively; $n$ is normal to the shell surface. Equations of the shell vibrations interacting with a fluid can be presented in the following operational form:

$$
\mathbf{L}(\Theta)+\mathbf{M}(\Theta)=\mathbf{P}
$$

where $\Theta=(u, v, w) ; \mathbf{L}(\Theta), \mathbf{M}(\Theta)$ are operators of elastic and mass forces, and $\mathbf{P}$ is a pressure drop. Linear flexural vibrations of the shell are presented in the following form: $w(x, t)=w(x)$ $\exp (i \omega t)$. Velocity circulation of the vibrating shell is determined from the singular integral equation:

$$
\frac{1}{4 \pi} \iint_{S} \Gamma(\varsigma) \frac{\partial^{2}}{\partial n_{x} \partial n_{\varsigma}}\left(\frac{1}{|x-\varsigma|}\right) d S_{\varsigma}=i \omega w(x)
$$

where $\Gamma(\varsigma)$ is the density of circulation; $n_{x}$ is the surface normal. The density of circulation describes the shell interaction with a fluid. The vortex method [70] is used to solve the singular integral equation. The fluid kinetic energy is taken into account in the following form:

$$
K=\frac{1}{2} \rho_{2} \int_{S} \Phi \frac{\partial \Phi}{\partial n_{x}} d S
$$

where $\rho_{2}$ is fluid density. As a result of a linear analysis, wet eigenmodes $W_{k}(x), U_{k}(x), V_{k}(x)$ are obtained numerically. Shell flexural vibrations $w(x, t)$ are expanded by using these eigenmodes and finite-degree-of-freedom dynamical system is obtained. Then the Shaw-Pierre NNMs are determined for this system. Results of the analysis are shown on the backbone curves. It is obtained that all backbone curves are hard. However, at a certain value of amplitudes, free nonlinear vibrations lose stability and NNMs become unstable at great vibrations amplitudes.

\section{Nonlinear Normal Modes of Continuous Systems}

Continuous nonlinear modes (CNMs) are used for direct solution of a distributed mechanical system. Theoretical investigations on this subject are considered in Refs. [1,3]. CNMs are divided into two types: Shaw-Pierre CNMs and King-Vakakis CNMs.

The Shaw-Pierre CNMs are suggested in Ref. [71]. These nonlinear modes are used to analyze flexural vibrations of a beam on a nonlinear base:

$$
w_{t}=v ; v_{t}=F(w(x, t), v(x, t))=-w_{x x x x}-k w-\gamma w^{3}
$$

The beam is considered simply supported:

$$
w(0, t)=w(1, t)=w_{x x}(0, t)=w_{x x}(1, t)=0
$$

CNMs are represented as two-dimensional invariant manifolds. These manifolds are touched on the proper subspace of linearized system in equilibrium and pass through equilibrium $(w, v)$ $=(0,0)$. CNMs take the following form:

$$
w(x, t)=U\left(w_{0}(t), v_{0}(t), x, x_{0}\right) ; \quad v(x, t)=V\left(w_{0}(t), v_{0}(t), x, x_{0}\right)
$$

where $w_{0}(t)=w\left(x_{0}, t\right) ; v_{0}(t)=v\left(x_{0}, t\right) ; U, V$ are certain functions. Dynamics of the system on CNM is described by a one-degree-offreedom nonlinear oscillator. CNMs are synchronous motions and they are a generalization of the Shaw-Pierre NNMs for a finitedegree-of-freedom system. Functions $U\left(w_{0}(t), v_{0}(t), x, x_{0}\right)$; $V\left(w_{0}(t), v_{0}(t), x, x_{0}\right)$ satisfy the following system of hyperbolic partial differential equations:

$$
\begin{aligned}
V & =\frac{\partial U}{\partial w_{0}} v_{0}+\frac{\partial U}{\partial v_{0}}[F(U, V)]_{X=X_{0}} \\
F(U, V) & =\frac{\partial V}{\partial w_{0}} v_{0}+\frac{\partial V}{\partial v_{0}}[F(U, V)]_{X=X_{0}}
\end{aligned}
$$

This system is completed with the boundary conditions in Eq. (81). 
Manifold (Eq. (82)) takes the form of asymptotic series:

$$
\begin{aligned}
U\left(w_{0}, v_{0}, x, x_{0}\right)= & a_{1}\left(x, x_{0}\right) w_{0}(t)+a_{2}\left(x, x_{0}\right) v_{0}(t) \\
& +a_{3}\left(x, x_{0}\right) w_{0}^{2}(t)+\ldots . \\
V\left(w_{0}, v_{0}, x, x_{0}\right)= & b_{1}\left(x, x_{0}\right) w_{0}(t)+b_{2}\left(x, x_{0}\right) v_{0}(t) \\
& +b_{3}\left(x, x_{0}\right) w_{0}^{2}(t)+\ldots .
\end{aligned}
$$

The asymptotic series in Eq. (84) are substituted into Eq. (83) and coefficients of $w_{0}^{m}(t) v_{0}^{m}(t) ; m, n=0,1,2, \ldots ; m+n \geq 1$ are equated. Functions $a_{i}(x), b_{i}(x)$ satisfy the system of linear ordinary differential equations. Solving this system, the continuous nonlinear mode is obtained:

$$
\begin{aligned}
& U=w_{0} a_{1}(x)\left(1-\gamma\left(a_{1}^{2}(x)-1\right) \frac{\left(-3 k w_{0}^{2}+37 n^{4} \pi^{4} w_{0}^{2}-3 v_{0}^{2}\right)}{320 n^{4} \pi^{4}\left(9 n^{4} \pi^{4}-k\right)}\right)+\cdots \\
& V=v_{0} a_{1}(s)\left(1-3 \gamma\left(a_{1}^{2}(s)-1\right) \frac{\left(-k w_{0}^{2}+39 n^{4} \pi^{4} w_{0}^{2}-v_{0}^{2}\right)}{320 n^{4} \pi^{4}\left(9 n^{4} \pi^{4}-k\right)}\right)+\cdots
\end{aligned}
$$

where $a_{1}(s)=\sin (n \pi x) / \sin \left(n \pi x_{0}\right)$. Motions on this CNM are described by the following one-degree-of-freedom oscillator:

$$
\begin{aligned}
& \ddot{w}_{0}+\left(n^{4} \pi^{4}+k\right) w_{0}-\frac{\gamma \sin ^{2}\left(\pi n x_{0}\right)}{16\left(9 n^{4} \pi^{4}-k\right)}\left(9 k-111 n^{4} \pi^{4}+4\left(k+n^{4} \pi^{4}\right)\right) w_{0}^{3} \\
& +\left(-9+12 \sin ^{2}\left(\pi n x_{0}\right)\right) w_{0} \dot{w}_{0}^{2}=0
\end{aligned}
$$

Vibrations of a cantilever beam with geometrical nonlinearity are studied in Ref. [72]. Motions of the beam are described by a nonlinear integro-partial-differential equation. The Shaw-Pierre CNMs are applied to analyze vibrations. This paper is the first work to employ the Shaw-Pierre CNMs in the study of "intrinsic" nonlinearities, which arise purely from large deformation kinematics. It is shown how the method can be adapted to handle nonlinear inertial operators in the equations of motions. It is demonstrated that nonlinearities in boundary conditions can have a significant influence on normal mode shapes.

The King-Vakakis CNMs are used to analyze vibrations of thin beams on nonlinear foundation in Ref. [73]. Nonlinear dynamics of this beam is described by the following partial differential equation with respect to dimensionless variables and parameters:

$$
w_{t t}+\varepsilon_{2} w_{x x x x}-w_{x x}+k_{1} w+\varepsilon_{1} w^{3}=0
$$

Equation (85) and its boundary conditions take the following operator form:

$$
w_{t t}=\Re^{(0)}[w]+\varepsilon_{1} \Re^{(1)}[w]+\varepsilon_{2} \Re^{(2)}[w] ; \quad B[w]=0
$$

where $\Re^{(i)}$ are differential operators; $B$ is the operator of the boundary conditions. The energy conservation law takes the following form:

$$
E=0.5 \int_{0}^{1} w_{t}^{2} d x+L^{(0)}[w]+\varepsilon_{1} L^{(1)}[w]+\varepsilon_{2} L^{(2)}[w]
$$

where $L^{(i)}[w]$ are integrodifferential operators. Synchronous motions of the flexible beam can be presented as:

$$
w(x, t)=W\left[x, w_{0}(t)\right]
$$

where $w_{0}(t)$ are displacements of certain beam points. Combining Eqs. (86) and (87), the following equation with respect to $W$ is derived:

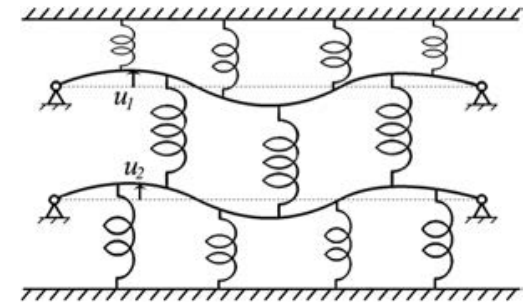

Fig. 14 Coupled beams on a nonlinear elastic foundation. Adopted from Ref. [74].

$$
\begin{aligned}
& 4 \frac{\partial^{2} W}{\partial w_{0}^{2}} \frac{E-L^{(0)}[W]-\varepsilon_{1} L^{(1)}[W]-\varepsilon_{2} L^{(2)}[W]}{\int_{0}^{1}\left[\frac{\partial W}{\partial w_{0}}\right]^{2} d x} \\
& \quad+\left.\frac{\partial W}{\partial w_{0}}\left\{\Re^{(0)}[W]+\varepsilon_{1} \Re^{(1)}[W]+\varepsilon_{2} \Re^{(2)}[W]\right\}\right|_{x=x_{0}} \\
& =\Re^{(0)}[W]+\varepsilon_{1} \Re^{(1)}\left[W, w_{0}\right]+\varepsilon_{2} \Re^{(2)}[W]
\end{aligned}
$$

The following condition is satisfied for analytical continuation of the solution up to maximal energetic surface:

$$
\begin{gathered}
{\left[\frac{\partial W}{\partial w_{0}}\left\{\Re^{(0)}[W]+\varepsilon_{1} \Re^{(1)}[W]+\varepsilon_{2} \Re^{(2)}[W]\right\}_{x=x_{0}}-\Re^{(0)}[W]\right.} \\
\left.\quad-\varepsilon_{1} \Re^{(1)}[W]-\varepsilon_{2} \Re^{(2)}[W]\right]_{w_{0}=w_{0}^{*}}=0
\end{gathered}
$$

Asymptotic series in terms of $\varepsilon_{1}$ powers is used to obtain CNM (Eq. (88)):

$$
W\left[x, w_{0}(t)\right]=\sum_{k=1}^{N} \varepsilon_{1}^{k} W_{k}\left(x, w_{0}(t)\right)
$$

Approach for determination of $W_{k}$ is considered in Ref. [73]. Results of CNM analysis are presented on the backbone curves, which are hard.

The King-Vakakis CNMs are used to analyze vibrations of coupled beams on a nonlinear elastic foundation (Fig. 14) in Ref. [74]. It is assumed that coupling between two beams is weak and the elastic base nonlinearity is small. System vibrations are described by the following system of partial differential equations:

$$
\begin{aligned}
& w_{1, t t}=-w_{1, x x x x}-k w_{1}-\varepsilon \gamma w_{1}^{3}-\varepsilon K\left(w_{1}-w_{2}\right) \\
& w_{2, t t}=-w_{2, x x x x}-k w_{2}-\varepsilon \gamma w_{2}^{3}-\varepsilon K\left(w_{2}-w_{1}\right)
\end{aligned}
$$

Localized CNMs are analyzed. It was shown that as the ratio of coupling over nonlinear terms tends to zero, strong nonlinear mode localization occurs in the system, where free oscillations were mainly confined to only one of two beams. Moreover, this strongly localized mode was found to be orbitally stable.

\section{Asymptotic Methods for NNMs Analysis}

Asymptotic methods are widely used in engineering sciences to calculate NNMs. Now applications of asymptotic methods for the NNMs analysis are considered.

5.1 Finite-Degree-of-Freedom Systems. A cyclic structure consisting of $n$ identical particles, arranged in a ring and interconnected by nonlinear springs is considered in Ref. [75]. These cyclic structures in linear approximation possess pair wise doubledegenerate natural frequencies with orthogonal natural modes. An asymptotic method is used to study a nonlinear interaction 
between pairs of modes with nearly identical natural frequencies. Subharmonic standing and traveling wave solutions are analyzed.

NNMs and their bifurcations in a mass-spring-pendulum system using perturbation methods and numerical procedure are studied in Ref. [76]. A structure of three beams with tip mass is treated in Ref. [77]. A six-degree-of-freedom model of this system is reduced through NNMs. System vibrations for 1:2 internal resonance are analyzed. Frequency responses of the system show the system behavior close to this resonance.

Vestroni et al. [78] considered a piecewise linear two-degreeof-freedom system:

$$
M \ddot{q}+\left[K_{0}-\varepsilon H(\eta) K_{2}\right] q=0
$$

where $M, K_{0}, K_{2}$ are second order square matrixes; $\eta=q_{2}-q_{1}$; $H(\eta)$ is the Heaviside function. The Lindstedt- Poincare asymptotic method is used to construct NNMs in the piecewise-linear system.

Self-sustained two-degree-of-freedom system under the action of external and parametric forces

$$
\begin{aligned}
& m_{1} \ddot{q}_{1}+f_{1}\left(\dot{q}_{1}\right)+\delta_{1} q_{1}+\gamma_{1} q_{1}^{3}+\left(\delta_{12}-\mu \cos 2 \omega t\right)\left(q_{1}-q_{2}\right) \\
& \quad=f \cos (\Omega t) \\
& m_{2} \ddot{q}_{2}+f_{2}\left(\dot{q}_{2}\right)+\delta_{2} q_{2}+\gamma_{2} q_{2}^{3}+\left(\delta_{12}-\mu \cos 2 \omega t\right)\left(q_{1}-q_{2}\right)=0
\end{aligned}
$$

is considered in Ref. [79]. The multiple scales method is used to obtain periodic motions of the system.

5.2 Dynamics of Beams and Shells. A one-dimensional continuous system with weak cubic geometrical and inertial nonlinearities is considered in Ref. [80]. This system is described by the following governing equation:

$$
\ddot{w}+\Im(w)+\aleph(w)=0
$$

where $\Im$ is the linear spatial operator; $\aleph$ is the nonlinear spatial and temporal operator. The system motions are expanded into a truncated series by spatial modes of vibrations. Generalized coordinates of this system are coefficients of this series terms. The Shaw-Pierre NNMs, the multiple scales method, and other approaches are used to analyze the obtained finite-degreeof-freedom system dynamics.

Nonlinear vibrations of beams with quadratic and cubic nonlinearities are treated in Ref. [81]. The equation of the beam vibrations with respect to dimensionless variables and parameters takes the form:

$$
w_{t t}+w_{x x x x}+\alpha_{1} w+\alpha_{2} w^{2}+\alpha_{3} w^{3}=0
$$

A multiple scales method is used to solve directly the partial differential equation. As a result, NNMs of the beam motions are obtained.

Fixed-fixed buckled beam vibrations in the neighborhood of its first post-buckling configuration are investigated in Ref. [82]. NNMs are computed by applying the method of multiple scales directly to the governing integro-partial-differential equation. Systems of modulation equations, which describe motions on the NNMs, are obtained. It is shown that for a three-to-one internal resonance between the first and the third modes, the beam may possess one stable uncoupled mode and either one stable coupled mode and three stable coupled modes or two stable and one unstable coupled modes. For the case of three-to-one internal resonances, the beam possesses either one unstable and three stable modes, or four stable modes or two stable and two unstable NNMs. For one-to-one internal resonance between the third and fourth modes, the beam possesses either six stable modes or one unstable and five stable modes or two unstable and four stable modes or four stable nonlinear modes.

Vertical beam vibrations under the action of seismic excitation are analyzed in Ref. [83]. Relations for nonlinear curvature and nonlinear inertia are taken into account in the beam model. The integro-partial-differential equation of the beam vibrations has the following form:

$$
\begin{aligned}
w_{t t} & +2 \varepsilon^{2} \mu w_{t}+w_{x x x x}+\left(w_{x}\left(w_{x} w_{x x}\right)_{x}\right)_{x} \\
& +0.5\left(w_{x} \int_{z_{n}}^{x} \frac{\partial^{2}}{\partial t^{2}}\left(\int_{0}^{s} w_{x}^{2} d x\right) d s\right)_{x}+\varepsilon^{2}\left[w_{x x}\left(x-z_{n}\right)+w_{x}\right] \\
& \times\left(a_{b} \cos (\Omega t)-g\right)-\varepsilon c w_{t}\left|w_{t}\right|
\end{aligned}
$$

The multiple scales method is used to solve directly the integropartial-differential equation in order to analyze the nonlinear vibrations. It is shown that the obtained motions correspond to the Kauderer-Rosenberg NNMs.

Beams coupled by nonlinear springs are investigated in Ref. [84]. Beam vibrations are expanded using the first two spatial eigenmodes. As a result, a two-degree-of-freedom nonlinear dynamical system is obtained. The method of averaging is used to analyze this system. It is shown that when the energy is slowly pumped into the system, the energy suddenly breaks and one of the local modes becomes the dominant energy receiver. Moreover, explicit expressions for the critical time at which onset of localization occurs is derived.

A system of two coupled cantilever beams with nonlinear grounding stiffness (Fig. 15) is considered in Ref. [85]. The Karhunen-Loeve decomposition procedure is used to obtain localized NNM. In order to apply this method, time series of displacements are obtained by the direct numerical integration of the partial differential equations. Bimodal interaction in buckled beams, shallow arches and suspended cables is analyzed in Ref. [86]. Nonlinear orthogonality of modes and activation of associated interactions are considered as dual problems. Properties of modes interaction in these elastic systems are analyzed. Number of coupled NNMs is determined.

A water-filled perfect circular cylindrical shell excited harmonically in the neighborhood of the fundamental frequency is treated in Ref. [87]. Keeping 16 natural modes, a finite-degree-offreedom system is obtained. A normal form procedure is applied to this system. The frequency response of the water-filled perfect shell is analyzed. A 16 degree-of-freedom system is reduced to a two-degree-of-freedom system. Thin shallow shells are considered in Ref. [88]. Shell displacements $u, v, w$ are expanded using trial functions and Lagrange equations are derived. Asymptotic approximations of NNMs are obtained using nonlinear forms of ordinary differential equations. Results of calculations are shown

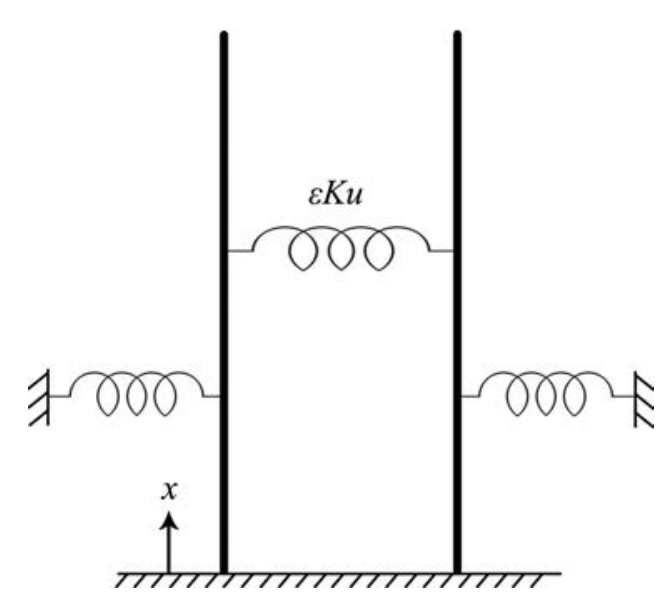

Fig. 15 System of two coupled beams. Adopted from Ref. [85]. 
on the frequency responses. Thin cylindrical water-filled shell is treated in Ref. [89]. A proper orthogonal decomposition method is used to extract the spatial behavior of the system. Temporal responses are obtained using the Galerkin procedure. NNMs are studied using normal forms of ordinary differential equations.

Nonlinear forced vibrations of a simply supported, circular cylindrical shell in a flow of incompressible, inviscid fluid are investigated using the Donnell's shallow shell theory [90,91]. Motions are obtained using normal forms for ordinary differential equations.

\section{Localization and Target Energy Transfer}

Principal aspects of the "targeted energy transfer" (TET) in light of NNMs theory are presented here. It means that TET is the irreversible transfer of the vibration energy from the subsystem of its generation to a nonlinear attachment. TET permits to damp undesirable large vibrations amplitudes of structures.

In general, different mechanisms of energy transfer exist. Nonlinear energy transfer is implemented due to nonlinear mode bifurcations, or through spatial energy localization by formation of NNMs [3,5,9,15-19,74,92-95]. In nonlinear Hamiltonian systems, the irreversible transfer of energy is generally precluded due to conservation of the phase volume. In certain cases, Hamiltonian dynamics can be trapped in bounded regions of the state space [96].

It is known that nonlinear interaction of vibrations modes often occurs due to internal resonances [97,98]. A new form of the nonlinear energy transfer between modes in harmonically forced structures is analyzed in Refs. [98-102]. This mechanism of passive energy transfer is caused by a resonance interaction of the slow modulation of a higher mode with a lower one. Kerschen et al. [103] discuss an alternative form of nonlinear modal interaction between NNMs with high energy. At low energies, these modes do not satisfy internal resonance conditions. However, at higher energies, the same NNMs become internally resonant, as their energy-dependent frequencies become closed and strongly nonlinear modal interactions. A similar phenomenon is observed in thin plate vibrations with geometrical nonlinearities [104]. A transient mode localization phenomenon in a system containing a simply supported beam and hardening nonlinear springs is considered by Pilipchuk [84]. An energy exchange between local modes is observed. Conditions of localization are obtained. It was shown that if the energy is slowly pumped into the system, one of these local modes become the dominant energy receiver.

Limiting phase trajectories (LPT) of mechanical systems with internal resonance are considered in Refs. [105,106]. LPT have the maximal energy exchange between partial oscillators in a form of beating.

Localization is one of the most interesting features of NNMs. In this case, certain substructures of a dynamical system have significant amplitudes of vibrations and the rest of the substructures have small amplitudes. Mode localization may occur in linear systems composed of coupled subsystems [107-109]. In nonlinear systems, structural mistuning is not a prerequisite for mode localization, since dependence of the oscillation frequency on the amplitude provides an 'effective mistuning' (or disorder) [3,110-112]. A nonlinear mode localization takes place in coupled oscillators either due to a bifurcation of similar NNMs in the symmetric system, or due to the energy dependence of the nonlinear mode shape of nonsimilar NNMs [5]. Forced nonlinear localization due to the normal mode bifurcation is studied in a twodegree-of-freedom oscillator with cubic stiffness nonlinearities [113]. A nonlinear mode localization in the system of coupled beams is analyzed in Refs. [114-116]. Localization in a flexible structure with nonsmooth nonlinearities is studied in Ref. [117]. A review of mode localization in systems governed by nonlinear partial differential equations is presented in Ref. [94]. Localized NNMs of the forced vibrations of systems consisting of a linear subsystem and essential nonlinear absorber are considered in
Refs. $[14,18,19,118]$. Different types of NNMs localization are treated for a low-dimensional cyclic system in Ref. [9]. It is obtained that the $N$-degree-of-freedom cyclic system possesses $N$ (strongly) localized NNMs, which are stable. From an engineering point of view, the nonlinear mode localization phenomena can be implemented in vibration isolation design [20].

Nonlinear TET in two-degree-of-freedom systems is discussed in several papers. This phenomenon is first treated by Gendelman [119] and Vakakis [120], who analyzed transients in a twodegrees-of-freedom system consisting of a damped linear subsystem and weakly coupled essentially nonlinear one-degreeof-freedom oscillator. Mechanical systems with slightly different nonlinear attachments are treated in Refs. [121,122]. Principal results on TET are presented in Ref. [5] and in a review [123], where it is shown that under certain conditions, a passive TET from a linear subsystem to a nonlinear energy sink (NES) occurs.

A transient resonance capture (TRC) in the resonance manifold takes place during certain time interval. This is considered as the main mechanism of the targeted energy transfer in coupled oscillators with strongly nonlinear attachments [5,121]. Methods of averaging, multiple scales and of complexification [124] are used in this investigation. Experimental verification of TET is presented in Refs. [5,125].

Now applications of NNMs theory to TET analysis are discussed. A degenerate bifurcation structure of NNMs in a linear oscillator coupled to a nonlinear attachment is studied by Gendelman et al. [126]. Vakakis and Rand [127] considered resonant dynamics of the Hamiltonian system at 1:1 internal resonance. They analyzed NNMs and elliptic orbits in configuration space. The invariant manifold approach is used to analyze NNMs of a linear subsystem coupled to one-degree-of-freedom nonlinear oscillator in Ref. [128]. Different types of TET were obtained. A method developed by Pilipchuk et al. [129] is used to study NNMs of a two-degree-of-freedom system with cubic nonlinearity [5]; NNMs at different resonances are considered. If the stable localized mode is excited by external forcing or by the initial conditions, then the system energy is rapidly transferred from the directly excited linear oscillator to the nonlinear attachment and a continuous exchange of energy between the linear subsystem and nonlinear attachment occurs in the form of beating.

TET is observed for main two-degree-of-freedom linear subsystem near 1:1:1 internal resonance manifold [130]. The nonlinear energy sink passively extracts energy from both modes of the main linear subsystem. TET phenomenon in a mechanical system with piecewise-quadratic nonlinear damping characteristics is treated in Ref. [131]. TET from the main linear subsystem to multidegree-of-freedom nonlinear energy sink is analyzed in Refs. [5,132-136]. However, system dynamics in TET is mainly determined by the corresponding Hamiltonian system.

TET in continuous mechanical systems is studied in Refs. [5,136-138]. These systems are composed of simply supported beams with attached nonlinear energy sink. These beams are forced impulsively. The nonlinear energy sink absorbs passively major portion of the beam energy. Dynamics of a plate with multidegree-of-freedom nonlinear energy sink is discussed in Ref. [139].

TET in systems containing harmonically forced single-degreeof-freedom linear oscillator and a nonlinear energy sink is treated in Refs. [140-143]. Steady states, weakly modulated responses, and a special type of strongly modulated response exist in these systems. It may be treated as an extension of TET to periodically forced structures. A possible application of nonlinear energy sinks as vibration absorbers for harmonically forced single- and multidegree-of-freedom linear subsystems is discussed. It is shown that under certain conditions a nonlinear energy sink can be an effective vibration absorber. Interaction of parametrically excited mechanical systems with a nonlinear energy sink is treated in Ref. [144].

In Ref. [5], vibro-impact nonlinear energy sinks are considered as shock absorbers. It is obtained that absorption mode can be 
achieved even for a small number of impacts. Impact energy sink is capable of absorbing significant portions of the energy of primary systems at a small time interval. Absorption of instabilities in drilling operations through passive TET are treated in Ref. [5].

\section{NNM Based Model Reduction}

NNMs allow for a reduction of the dimensions of the finitedegree-of-freedom nonlinear systems. In particular, motions on the modal lines of the Kauderer-Rosenberg NNMs in configuration space are described by one degree of freedom nonlinear oscillators. If the Shaw-Pierre NNMs are considered, equations of motions are projected on the corresponding NNM manifold. As a result of such projection for a system without internal resonances, one degree of freedom oscillator is obtained.

Specific construction of reduced-order models using NNMs is carried out in few papers. Reduced order models of geometrically nonlinear vibrations of thin-walled structures are computed for a general class of nonlinear systems with quadratic and cubic nonlinearities by Touze and Amabili [87]. NNMs are used to derive a reduced-order model for large amplitude, geometrically nonlinear vibrations of thin shells in Ref. [88]. Three different shell problems are considered: (i) simply supported doubly curved shallow shell, (ii) simply supported circular cylindrical panel, and (iii) simply supported, closed cylindrical shell. The proper orthogonal decomposition and asymptotic approximation of the NNMs are compared to reduce the complicated dynamics exhibited by large amplitude, geometrically nonlinear vibrations of thin shells by Amabili and Touze [89]. A water-filled, simply supported circular cylindrical shell subjected to harmonic excitation is treated. Both proper orthogonal decomposition and NNMs significantly reduce the dimension of the original model.

Order reduction of nonlinear systems with time periodic coefficients is considered by Sinha et al. [145]. Equations of motions are transformed using the Lyapunov-Floquet theory. Nonlinear order reduction methodology is also suggested through a generalization of the invariant manifold technique via time periodic center manifold theory. An approach for the model reduction based on the invariant manifold construction is discussed in Ref. [146]. Nonlinear dynamics on these manifolds is governed by few ordinary differential equations. Geometry of the manifold varies in time due to existence of external and parametric excitations. Governing partial differential equations for these manifolds are nonlinear with time-varying coefficients. Solutions of these equations are obtained using the Taylor and Fourier series.

Gabale and Sinha [147] consider a methodology for reduced order controller design for nonlinear dynamical systems. A proper coordinate transformation and state feedback can be found under which reduced order systems are transformed into linear, timeperiodic and closed-loop systems. This permits the design of a time-varying feedback controller, which guarantees stability of the system.

Proper orthogonal decomposition and the nonlinear Galerkin method are used to reduce infinite dimensional systems to low dimensional finite degree of freedom systems by Steindl and Troger [148].

Assembly of nonlinear component structures is considered in Ref. [149]. The methodology to obtain reduced-order dynamic models is described. This approach is a nonlinear extension of component mode synthesis technique developed for linear structures. NNMs are constructed for substructures using invariant manifold approach. The proposed method is applicable to largescaled nonlinear structural systems, which are obtained by the finite element method.

\section{Other References of NNMs}

Planar motions of a rigid body with linear springs and skatelike nonholonomic constraints are considered in Ref. [150]. This system is reduced to a 2.5 degree-of-freedom system. Using the method of averaging, two families of NNMs are obtained.

Dynamics of a conservative spherical pendulum is studied in Ref. [151]. The system dominant dynamics is slow and it is realized as a three-dimensional manifold in the phase-space. A three-dimensional slow invariant manifold contains twodimensional submanifold, which is the NNM. The master slow dynamics is described by a single nonlinear oscillator.

NNMs and band zones in finite granular chains composed of spherical granular beads in Hertzian contacts are treated in Ref. [152]. The system has NNMs that appear as traveling waves. This paper provides a classification of coherent intrinsic dynamics of one-dimensional homogeneous granular chains with no precompression. Traveling waves in nonlinear chains and beams, as a generalization of NNMs, are considered in Ref. [153]. A passage to a long wavelength approximation is made. Conditions of a solitary wave existence are obtained. Waves with regard to elastic impacts have been investigated.

Numerical calculations of NNMs in multidegree-of-freedom structural models are considered in Ref. [154].

\section{Conclusions}

Different applications of NNMs are considered in this review. The Kauderer-Rosenberg and Shaw-Pierre concepts of the NNMs and other asymptotical approaches are used to determine these regimes and to describe important properties of different mechanical systems. The Kauderer-Rosenberg NNMs are an effective tool to study free and forced large amplitude vibrations of low dimensional essential nonlinear systems without dissipation. The Shaw-Pierre NNMs are used to investigate free and forced nonlinear vibrations of mechanical systems with dissipation. Nonlinear dynamics of finite-degree-of-freedom mechanical systems, such as rotors, car suspension, systems with the essentially nonlinear absorbers etc., can be successfully analyzed using NNMs. Both Kauderer-Rosenberg and Shaw-Pierre NNMs can be used to study dynamics of rods, blades, arches, plates, and shells. Frequency responses and other dynamical characteristics are obtained using the NNMs approach or by a combination of this approach with other methods. In particular, a combination of the NNMs theory and the Rauscher method is effective to describe resonance vibrations in nonlinear systems. Moreover, the NNMs approach is effective not only in analysis of smooth systems, but for the nonsmooth systems dynamics as well. NNMs play a key role in investigation of localization and energy transfer. NNM investigation is an important step in analyzing the global behavior of nonlinear mechanical systems. NNMs can be considered as fundamental motions, which allow to determine more complicated behavior of nonlinear systems.

\section{References}

[1] Mikhlin, Y, and Avramov, K. V., 2010 "Nonlinear Normal Modes for Vibrating Mechanical Systems. Review of Theoretical Developments," ASME Appl Mech. Rev., 63(6), p. 060802.

[2] Manevich, L. I., Mikhlin, Y., and Pilipchuk, V. N., 1989, The Method of Normal Oscillation for Essentially Nonlinear Systems, Nauka, Moscow (in Russian)

[3] Vakakis, A., Manevitch, L., Mikhlin, Y., Pilipchuk, V., and Zevin, A., 1996, Normal Modes and Localization in Nonlinear Systems, Wiley, New York.

[4] Avramov, K. V., and Mikhlin, Y., 2010, Nonlinear Dynamics of Elastic Systems: Models, Methods and Approaches, Vol. 1, Scientific Centre "Regular and Chaotic Dynamics", Moscow (in Russian).

[5] Vakakis, A. F., Gendelman, O. V., Bergman, L. A., McFarland, D. M. Kerschen, G., and Lee Y. S., 2008, Nonlinear Targeted Energy Transfer in Mechanical and Structural Systems (Solid Mechanics and Its Applications), Vol. 156, Springer, New York.

[6] Pilipchuk, V. N., 2010, Nonlinear Dynamics: Between Linear and Impact Limits, Springer-Verlag, Berlin.

[7] Amabili, M., 2008, Nonlinear Vibrations and Stability of Shells and Plates, Cambridge University Press, New York.

[8] Leissa, A. W., 1993, "Vibration of Shells," NASA Report No. SP-288, Government Printing Office, Washington, DC. 
[9] Vakakis, A. F., and Cetinkaya, C., 1993, "Mode Localization in a Class of Multi Degrees-of-Freedom Nonlinear Systems With Cyclic Symmetry," SIAM J. Appl. Math., 35, pp. 265-282.

[10] Mikhlin, Y., Vakakis, A. F., and Salenger, G., 1998, "Direct and Inverse Problems Encountered in Vibro-Impact Oscillations of a Discrete System," J. Sound Vib., 216, pp. 227-250.

[11] Vedenova, E., Manevich, L., and Pilipchuk, V., 1985, "Normal Oscillations of a String With Concentrated Masses on Non-Linearly Elastic Support," Prikl Matem i Mekh (PMM USSR), 49, pp. 203-211.

[12] Mikhlin, Y., and Morgunov, B. I., 2001, "Normal Vibrations in NearConservative Self-Excited and Viscoelastic Nonlinear Systems," Nonlinear Dyn., 25, pp. 33-48.

[13] Peeters, M., Kerschen, G., and Golinval, J. C., 2011, "Dynamic Testing of Nonlinear Vibrating Structures Using Nonlinear Normal Modes," J. Sound Vib., 330, pp. 486-509.

[14] Mikhlin, Y., and Reshetnikova, S. N., 2005, "Dynamical Interaction of an Elastic System and Essentially Nonlinear Absorber," J. Sound Vib., 283, pp. 91-120.

[15] Mikhlin, Y., and Reshetnikova, S. N., 2006, "Dynamical Interaction of an Elastic System and an Vibro-Impact Absorber," Math. Probl. Eng., 2006, p. 37980.

[16] Mikhlin, Y., and Reshetnikova, S. N., 2004, "Analysis of Some Problems of the Elastic Vibrations Absorption by Using the Vibro-Impact Oscillators," Dynamics and Strength of Machines, Vol. 20, O. K. Morachkovsky, ed., National Technical University "KhPI", Kharkiv, Ukraine, pp. 55-64 (in Russian).

[17] Avramov, K. V., and Mikhlin, Y., 2004, "Snap-Through Truss as a Vibration Absorber," J. Vib. Control, 10, pp. 291-308.

[18] Avramov, K. V., and Mikhlin, Y., 2004, "Forced Oscillations of a System Containing a Snap-Through Truss, Close to Its Equilibrium Position," Nonlinear Dyn., 35, pp. 361-379.

[19] Avramov, K. V., and Mikhlin, Y., 2006, "Snap-Through Truss as an Absorber of Forced Oscillations," J. Sound Vib., 290, pp. 705-722.

[20] Jiang, X., and Vakakis, A. F., 2003, "Dual Mode Vibration Isolation Based on Non-Linear Mode Localization,” Int. J. Nonlinear Mech., 37, pp. 837-850.

[21] Breslavsky, I., Avramov, K. V., Mikhlin, Y., and Kochurov, R., 2008, "Nonlinear Modes of Snap-Through Motions of a Shallow Arch," J. Sound Vib., 311, pp. 297-313.

[22] Avramov, K. V., and Gendelman, O. V., 2010, "On Interaction of Vibrating Beam With Essentially Nonlinear Absorber,” Mecanica, 45, pp. 355-365.

[23] Chati, M., Rand, R., and Mukherjee, S., 1997, "Modal Analysis of a Cracked Beam,” J. Sound Vib., 207, pp. 249-270.

[24] Donnell, L. H., 1934, "A New Theory for the Buckling of Thin Cylinders Under Axial Compression and Bending," Trans. ASME, 56, pp. 795-806.

[25] Avramov, K. V., and Mikhlin, Y., 2002, "Analysis of Nonlinear Normal Modes in Cylindrical Shells," Nonlinear Dynamics of Shells With Fluid-Structure Interaction, NATO CLG Grant Report Proceedings, F. Pellicano, Y. Mikhlin, and I. Zolotarev, eds., Institute of Thermomechanics, Prague, pp. 33-44.

[26] Avramov, K. V., Mikhlin, Y., and Kurilov, E., 2007, "Asymptotic Analysis of Nonlinear Dynamics of Simply Supported Cylindrical Shells," Nonlinear Dyn., 47, pp. 331-352.

[27] Kubenko, V. D., Koval'shuk, P. S., and Krasnopolskaya, T. S., 1984, Nonlin ear Interaction of Modes of Bending Shell Oscillations, Naukova Dumka, Kiev (in Russian).

[28] Avramov, K. V., 2012, "Nonlinear Modes of Vibrations for Simply Supported Cylindrical Shell With Geometrical Nonlinearity," Acta Mech., 223, pp. 279-292.

[29] Avramov, K. V., 2011, "Many-Dimensional Models of Traveling Waves and Nonlinear Modes in Cylindrical Shell," Int. Appl. Mech., 47, pp. 70-77.

[30] Kochurov, R., and Avramov, K. V., 2010, "Nonlinear Modes and Traveling Waves of Parametrically Excited Cylindrical Shells," J. Sound Vib., 329, pp. 2193-2204.

[31] Kochurov, R., and Avramov, K. V., 2011, "Parametric Vibrations of Cylindrical Shells Subject to Geometrically Nonlinear Deformation: Multimode Models," Int. Appl. Mech., 46, pp. 1010-1018.

[32] Mikhlin, Y., and Kurilov, E., 2009, "On Nonlinear Vibrations of Cylindrical Shells in Supersonic Flow," J. Mech. Eng., 12, pp. 52-58 (in Russian).

[33] Kurilov, E., and Mikhlin, Y., 2007, "Nonlinear Vibrations of Cylindrical Shells With Initial Imperfections in a Supersonic Flow," Int. Appl. Mech., 43, pp. $1000-1008$.

[34] Shaw, S. W., and Pierre, C., 1993, "Normal Modes for Non-Linear Vibratory Systems," J. Sound Vib., 164, pp. 85-124.

[35] Pesheck, E., Pierre, C., and Shaw, S. W., 2002, “A New Galerkin-Based Approach for Accurate Non-Linear Normal Modes Through Invariant Manifolds," J. Sound Vib., 249, pp. 971-993.

[36] Jiang, D., Pierre, C., and Shaw, S. W., 2005, "Nonlinear Normal Modes for Vibratory Systems Under Harmonic Excitation," J. Sound Vib., 288, pp. 791-812.

[37] Falzarano, J. M., Clague, R. E., and Kota, R. S., 2001, “Application of Nonlinear Normal Mode Analysis to the Nonlinear and Coupled Dynamics of a Floating Offshore Platform With Damping," Nonlinear Dyn., 25, pp. 255-274.

[38] Mikhlin, Y., and Mitrokhin, S., 2011, "Nonlinear Oscillatory Processes in Wheeled Vehicles," Int. Appl. Mech., 46, pp. 1311-1318.

[39] Mikhlin, Y., and Perepelkin, N. V., 2011, "Nonlinear Normal Modes and Their Applications in Mechanical Systems," Proc. Inst. Mech. Eng., Part C, 225, pp. $2369-2384$.

[40] Villa, C. V. S., Sinou, J. J., Thouverez, F., 2005, "The Invariant Manifold Approach Applied to Nonlinear Dynamics of a Rotor-Bearing System," Eur. J. Mech. A/Solids, 24, pp. 676-689.
[41] Avramov, K. V., and Borisuk, A., 2011, "Nonlinear Dynamics of One Disk Asymmetrical Rotor Supported by Two Journal Bearings," Nonlinear Dyn., 67, pp. 1201-1219.

[42] Avramov, K. V., 2010, "A Model of Asymmetric Single-Disk Rotor SelfVibrations," Strength Mater, 42, pp. 459-470.

[43] Perepelkin, N. V., and Mikhlin, Y., 2011, "Analysis of Forced Modes of OneDisk Rotors on Nonlinear Elastic Base,” Proc. Inst. Appl. Math. Mech.: Mech. Solids, 40, pp. 221-232 (in Russian).

[44] Perepelkin, N. V., and Mikhlin, Y., 2011, "Construction and Analysis of Stability of Resonance Regimes of the One-Disk Rotor Motion on Nonlinear Flexible Supports,” Dyn. Systems, 29(2), pp. 269-280 (in Russian). Available at http://dynsys.crimea.edu/issue/29_2/dynsys_29_2_perepelkin.pdf

[45] Perepelkin, N. V., and Mikhlin, Y., 2011, "Nonlinear Normal Modes of Forced Vibrations in Rotor Systems," Proceedings of the 7th European Nonlinear Dynamics Conference, Roma, Italy.

[46] Avramov, K. V., 2008, "Analysis of Forced Vibrations by Nonlinear Modes," Nonlinear Dyn., 53, pp. 117-127.

[47] Warminski, J., 2008, "Nonlinear Normal Modes of Coupled Self-Excited Oscillators in Regular and Chaotic Vibration Regimes," J. Theor. Appl Mech., 46, pp. 693-714. Available at http://ptmts.org.pl/Warminski-3-08.pdf

[48] Chen, S. L., and Shaw, S. W., 1996, "Normal Modes for Piecewise Linear Vibratory Systems," Nonlinear Dyn., 10, pp. 135-164.

[49] Jiang, D., Pierre, C., and Shaw, S. W., 2004, "Large-Amplitude Non-Linear Normal Modes of Piecewise Linear Systems," J. Sound Vib., 272, pp. 869-891.

[50] Boivin, N., Pierre, C., and Shaw, S. W., 1995, "Non-Linear Normal Modes, Invariance, and Modal Dynamics Approximations of Non-Linear Systems," Nonlinear Dyn., 8, pp. 315-346. Available at http://deepblue.lib.umich.edu/bitstream/ handle/2027.42/43333/11071 2004_Article BF00045620.pdf;jsessionid=078F066 77999DA229F323481CFDBB5AD? sequence=1

[51] Pesheck, E., Boivin, N., and Pierre, C., 2001, "Nonlinear Modal Analysis of Structural Systems Using Multi-Mode Invariant Manifolds," Nonlinear Dyn., 25, pp. 183-205.

[52] Pesheck, E., Pierre, C., and Shaw, S. W., 2001, "Accurate Reduced-Order Models for a Simple Rotor Blade Model Using Nonlinear Normal Modes," Math Comput. Model., 33, pp. 1085-1097.

[53] Pesheck, E., Pierre, C., and Shaw, S. W., 2002, "Modal Reduction of a Nonlinear Rotating Beams Through Nonlinear Normal Modes," ASME J.Vib. Acoust., 124(2), pp. 229-236.

[54] Jiang, D., Pierre, C., and Shaw, S. W., 2005, "The Construction of Non-Linear Normal Modes for Systems With Internal Resonances," Int. J. Nonlinear Mech., 40, pp. 729-746.

[55] Xie, W. C., Lee, H. P., and Lim, S. P., 2002, "Normal Modes of a Non-Linear Clamped-Clamped Beam," J. Sound Vib., 250, pp. 339-349.

[56] Avramov, K. V., Pierre, C., and Shyriaieva, N., 2007, "Flexural-Flexural-Torsional Nonlinear Vibrations of Pre-Twisted Rotating Beams With Asymmetric Cross-Sections," J. Vib. Control, 13, pp. 329-364.

[57] Avramov, K. V., Galas, O. S., and Morachkovskii, O. K., 2009, “Analysis of Flexural-Flexural-Torsional Nonlinear Vibrations of Twisted Rotating Beams With Cross-Sectional Deplanation," Strength Mater., 41, pp. 200-208.

[58] Avramov, K. V., 2009, "Nonlinear Modes of Parametric Vibrations and Their Applications to Beams Dynamics," J. Sound Vib., 322, pp. 476-489.

[59] Touze, C., Thomas, O., and Chaigne, A., 2004, "Hardening/Softening Behaviour in Non-Linear Oscillations of Structural Systems Using Non-Linear Normal Modes," J. Sound Vib., 273, pp. 77-101.

[60] Li, X., Chen, Y., Wu, Z., and Chen, F., 2002, "Bifurcation of Nonlinear Internal Resonant Normal Modes of a Class of Multi-Degrees-Of-Freedom Systems," Mech. Res. Commun., 29, pp. 299-306.

[61] Soares, M. E. S., and Mazzilli, C. E. N., 2000, "Nonlinear Normal Modes of Planar Frames Discretised by the Finite Element Method," Comput. Struct., 77, pp. 485-493.

[62] Legrand, M., Jiang, D., Pierre, C., and Shaw, S. W., 2004, "Nonlinear Norma Modes of a Rotating Shaft Based on the Invariant Manifold Method," Int. J. Rotating Mach., 10, pp. 319-335.

[63] Mazzilli, C. E. N., Monticelli, G. C., and Galan Neto, N. A., 2011, "ReducedOrder Modeling in Non-Linear Dynamics: An Approach Based on Non-Linear Modes," Proc. Inst. Mech. Eng., Part C, 225, pp. 2354-2368.

[64] Avramov, K. V., and Strel'nikova, E. A., 2011, "Nonlinear Normal Modes of Self-Sustained Vibrations of Finite-Degrees-of-Freedom Mechanical Systems," Not. Nat. Acad. Sci. Ukraine, 2, pp. 44-51 (in Russian). Available at http://www.nbuv.gov.ua/portal/All/reports/2011-02/11-02-07.pdf

[65] Breslavsky, I., and Avramov, K. V., 2011, "Nonlinear Modes of Cylindrical Panels With Complex Boundaries. R-Function Method," Meccanica, 46, pp. 817-832.

[66] Breslavsky, I. D., and Avramov, K. V., 2010, "Vibrations of a ComplexShaped Panel," Int. Appl. Mech., 46, pp. 580-587.

[67] Rvachev, V. L., 1982, R-Functions Theory and Some of Its Applications, Naukova Dumka, Kiev (in Russian).

[68] Avramov, K. V., Tyshkovets, O., and Maksymenko-Sheyko, K. V., 2010, "Analysis of Nonlinear Free Vibration of Circular Plates With Cut-Outs Using R-Function Method," ASME J. Vib. Acoust., 132(5), p. 051001.

[69] Breslavsky, I. D., Strel'nikova, E. A., and Avramov, K. V., 2011, "Dynamics of Shallow Shells With Geometrical Nonlinearity Interacting With Fluid," Comput. Struct., 89, pp. 496-506.

[70] Belotserkovskii, S. M., 1977, "Study of the Unsteady Aerodynamics of Lifting Surfaces Using the Computer," Ann. Rev. Fluid Mech., 9, pp. 469-494.

[71] Shaw, S., and Pierre, C., 1994, "Normal Modes of Vibration for Non-Linear Continuous Systems," J. Sound Vib., 169, pp. 319-347. 
[72] Hsieh, S. R., Shaw, S. W., and Pierre, C., 1994, "Normal Modes for Large Amplitude Vibration of a Cantilever Beam,” Int. J. Solids Struct., 31, pp. 1981-2014.

[73] Pellicano, F., and Vakakis, A. F., 2001, "Normal Modes and Boundary Layers for a Slender Tensioned Beam on a Nonlinear Foundation," Nonlinear Dyn., 25, pp. 79-93.

[74] King, M. E., and Vakakis, A. F., 1995, "Asymptotic Analysis of Nonlinear Mode Localization in a Class of Coupled Continuous Structures," Int. J. Soilds Struct., 32, pp. 1161-1177.

[75] Samaranayake, S., and Bajaj, A. K., 1997, "Subharmonic Oscillations in Harmonically Excited Mechanical Systems With Cyclic Symmetry," J. Sound Vib., 206, pp. 39-60.

[76] Wang, F., Bajaj, A., and Kamiya, K., 2005, "Nonlinear Normal Modes and Their Bifurcations for an Inertially Coupled Nonlinear Conservative System," Nonlinear Dyn., 42, pp. 233-265.

[77] Wang, F., Bajaj, A. K., 2011, "Model Reduction for Discrete and Elastic Structures With Internal Quadratic Non-Linearities,” Proc. Inst. Mech Eng., Part C, 225, pp. 2422-2435.

[78] Vestroni, F., Luongo, A., and Paolone, A., 2008, “A Perturbation Method for Evaluating Nonlinear Normal Modes of a Piecewise Linear Two-Degrees-ofFreedom System," Nonlinear Dyn., 54, pp. 379-393.

[79] Warminski, J., 2010, "Nonlinear Normal Modes of a Self-Excited System Driven by Parametric and External Excitation," Nonlinear Dyn., 61, pp. 677-689.

[80] Nayfeh, A. H., and Nayfeh, S. A., 1994, "On Nonlinear Modes of Continuous Systems," ASME J. Vib. Acoust., 116(1), pp. 129-136.

[81] Nayfeh, A. H., and Nayfeh, S. A., 1995, "Nonlinear Normal Modes of a Continuous System With Quadratic Nonlinarities," ASME J. Vib. Acoust., 117(2), pp. 199-205.

[82] Nayfeh, A. H., Lacarbonara, W., and Chin, C. M., 1999, "Nonlinear Normal Modes of Buckled Beams: Three-to-One and One-to-One Internal Resonances," Nonlinear Dyn., 18, pp. 253-273.

[83] Yabuno, H., and Nayfeh, A. H., 2001, "Nonlinear Normal Modes of a Parametrically Excited Cantilever Beam," Nonlinear Dyn., 25, pp. 65-77.

[84] Pilipchuk, V. N., 2009, "Transition From Normal to Local Modes in an Elastic Beam Supported by Nonlinear Springs,” J. Sound Vib., 322, pp. 554-563.

[85] Ma, X., Azeez, M. F. A., and Vakakis, A. F., 2000, "Non-Linear Norma Modes and Non-Parametric System Identification of Non-Linear Oscillators," Mech. Syst. Signal Process., 14, pp. 37-48.

[86] Lacarbonara, W., and Rega, G., 2003, "Resonant Non-Linear Normal Modes. Part II: Activation/Orthogonality Conditions for Shallow Structural Systems," Int. J. Nonlinear Mech., 38, pp. 873-887.

[87] Touze, C., and Amabili, M., 2006, "Nonlinear Normal Modes for Damped Geometrically Nonlinear Systems: Applications to Reduced-Order Modelling of Harmonically Forced Structures," J. Sound Vib., 298, pp. 958-981.

[88] Touze, C., Amabili, M., and Thomas, O., 2008, "Reduced-Order Models for Large-Amplitude Vibrations of Shells Including In-Plane Inertia," Comput. Methods Appl. Mech. Eng., 197, pp. 2030-2045.

[89] Amabili, M., and Touze, C., 2007, "Reduced-Order Models for Nonlinear Vibrations of Fluids-Filled Circular Cylindrical Shells: Comparison of POD and Asymptotic Nonlinear Normal Modes Methods," J. Fluids Struct., 23, pp. 885-903.

[90] Pellicano, F., Amabili, M., and Vakakis, A. F., 2000, "Nonlinear Vibrations and Multiple Resonances of Fluid-Filled, Circular Shells. Part 2: Perturbation Analysis," ASME J. Vib. Acoust., 122(4), pp. 355-364.

[91] Amabili, M., Pellicano, F., Paidoussis, M. P., 1998, "Nonlinear Vibrations of Simply Supported, Circular Cylindrical Shells, Coupled to Quiescent Fluid," J. Fluids Struct., 12, pp. 883-918.

[92] King, M. E., and Vakakis, A. F., 1996, "An Energy-Based Approach to Computing Resonant Nonlinear Normal Modes,” ASME J. Appl. Mech., 63(3), pp. 810-819.

[93] Boivin, N., Pierre, C., and Shaw, S. W., 1995, "Nonlinear Modal Analysis of Structural Systems Featuring Internal Resonances,” J. Sound Vib., 182, pp. 336-341.

[94] Vakakis, A. F., 1996, "Nonlinear Mode Localization in Systems Governed by Partial Differential Equations," ASME Appl. Mech. Rev., 49, pp. 87-99.

[95] Lacarbonara, W., Rega, G., and Nayfeh, A. H., 2003, "Resonant Nonlinear Normal Modes, Part I: Analytical Treatment for Structural One Dimensional Systems,” Int. J. Nonlinear Mech., 38, pp. 851-872.

[96] Zaslavskii, G. M., 2005, Hamiltonian Chaos and Fractional Dynamics, Oxford University Press, Oxford.

[97] Nayfeh, A. H., and Mook, D. T., 1995, Nonlinear Oscillations, John Wiley \& Sons, New York.

[98] Nayfeh, A. H., 2000, Nonlinear Interactions: Analytical, Computational and Experimental Methods, Wiley Interscience, New York.

[99] Nayfeh, S. A., and Nayfeh, A. H., 1994, "Energy Transfer From High- to Low-Frequency Modes in a Flexible Structure via Modulation,” ASME J. Vib. Acoust., 116(2), pp. 203-207.

[100] Nayfeh, A. H., and Mook, D. T., 1995, "Energy Transfer From HighFrequency to Low-Frequency Modes in Structures," ASME J. Vib. Acoust., 117(B), pp. 186-195.

[101] Oh, K., and Nayfeh, A. H., 1998, "High- to Low-Frequency Modal Interactions in a Cantilever Composite Plate," ASME J. Vib. Acoust., 120(2), pp. 579-587.

[102] Malatkar, P., and Nayfeh, A. H., 2003, "On the Transfer of Energy Between Widely Spaced Modes in Structures," Nonlinear Dyn., 31, pp. 225-242.

[103] Kerschen, G., Peeters, M., Golinval, J. C., and Vakakis, A. F., 2009, "Nonlinear Normal Modes, Part I: A Useful Framework for the Structural Dynamics," Mech. Syst. Signal Process., 23, pp. 195-216.
[104] Breslavsky, I. D., and Avramov, K. V., 2012, "Two Modes Nonresonance Interaction for Rectangular Plate With Geometrical Non-Linearity," Nonlinear Dyn., 69, pp. 285-294

[105] Manevitch, L. I., 2007, "New Approach to Beating Phenomenon in Coupled Nonlinear Oscillatory Chain," Arch. Appl. Mech., 77, pp. 301-312.

[106] Manevitch, L. I., and Gendelman, O. V., 2011, Tractable Models of Solid Mechanics, Springer, Berlin.

[107] Anderson, P. W., 1958, "Absence of Diffusion in Certain Random Lattices," Phys. Rev., 109, pp. 1492-1505.

[108] Pierre, C., and Dowell, E. H., 1987, "Localization of Vibrations by Structura Irregularity," J. Sound Vib., 114, pp. 549-564.

[109] Hodges, C. H., 1982, "Confinement of Vibration by Structural Irregularity," J. Sound Vib., 82, pp. 411-424.

[110] Vakakis, A. F., Nayfeh, A. H., and King, M. E., 1993, "A Multiple-Scales Analysis of Nonlinear, Localized Modes in a Cyclic Periodic System," ASME J. Appl. Mech., 60(2), pp. 388-397.

[111] Vakakis, A. F., King, M. E., and Pearlstein, A. J., 1994, "Forced Localization in a Periodic Chain of Nonlinear Oscillators," Int. J. Nonlinear Mech., 29, pp. $429-447$.

[112] King, M. E., and Vakakis, A. F., 1995, "Mode Localization in a System of Coupled Flexible Beams With Geometric Nonlinearities," ZAMM, 75, pp. 127-139.

[113] Vakakis, A. F., 1992, "Fundamental and Subharmonic Resonances in a System With a 1-1 Internal Resonance," Nonlinear Dyn., 3, pp. 123-143.

[114] Vakakis, A. F., 1994, "Passive Spatial Confinement of Impulsive Responses in Coupled Nonlinear Beams," AIAA J., 32, pp. 1902-1910.

[115] Aubrecht, J., and Vakakis, A. F., 1996, "Localized and Non-Localized Nonlinear Normal Modes in a Multi-Span Beam With Geometric Nonlinearities," ASME J. Vib. Acoust., 118(4), pp. 533-542.

[116] Aubrecht, J., Vakakis, A. F., Tsao, T. C., and Bentsman, J., 1996 "Experimental Study of Nonlinear Transient Motion Confinement in a System of Coupled Beams," J. Sound Vib., 195, pp. 629-648.

[117] Emaci, E., Nayfeh, T. A., and Vakakis, A. F., 1997, "Numerical and Experimental Study of Nonlinear Localization in a Flexible Structure With VibroImpacts," ZAMM, 77, pp. 527-541.

[118] Avramov, K. V., and Gendelman, O. V., 2009, "Interaction of Elastic System With Snap-Through Vibration Absorber," Int. J. Nonlinear Mech., 44, pp. $81-89$.

[119] Gendelman, O. V., 2001, "Transition of Energy to a Nonlinear Localized Mode in a Highly Asymmetric System of Two Oscillators," Nonlinear Dyn., 25, pp. 237-253.

[120] Vakakis, A. F., 2001, "Inducing Passive Nonlinear Energy Sinks in Vibrating Systems," ASME J. Vib. Acoust., 123(3), pp. 324-332.

[121] Vakakis, A. F., and Gendelman, O., 2001, "Energy Pumping in Nonlinear Mechanical Oscillators: Part II-Resonance Capture," ASME J. Appl. Mech. 68(1), pp. 42-48.

[122] Gendelman, O. V., Manevitch, L. I., Vakakis, A. F., and McCloskey, R., 2001, "Energy Pumping in Nonlinear Mechanical Oscillators: Part I-Dynamics of the Underlying Hamiltonian Systems," ASME J. Appl. Mech., 68(1), pp. 34-41.

[123] Gendelman, O., 2011, "Targeted Energy Transfer in Systems With External and Self-Excitation," Proc. Inst. Mech. Eng., Part C, 225, pp. 2007-2043.

[124] Manevitch, L. I., 1999, "Complex Representation of Dynamics of Coupled Nonlinear Oscillators," Mathematical Models of Non-Linear Excitations, Transfer, Dynamics, and Control in Condensed Systems and Other Media, Kluwer Academic/Plenum Publishers, New York, pp. 269-300.

[125] McFarland, D. M., Kerschen, G., Kowtko, J. J., Lee, Y. S., Bergman, L. A., and Vakakis, A. F., 2005, "Experimental Investigation of Targeted Energy Transfer in Strongly and Nonlinearly Coupled Oscillators," J. Acoust. Soc. Am., 118, pp. 791-799.

[126] Gendelman, O. V., Manevitch, L. I., Vakakis, A. F., and Bergman, L. A., 2003 , "A Degenerate Bifurcation Structure in the Dynamics of Coupled Oscillators With Essential Stiffness Nonlinearities," Nonlinear Dyn., 33, pp. 1-10.

[127] Vakakis, A. F., and Rand, R. H., 2004, "Non-Linear Dynamics of a System of Coupled Oscillators With Essential Stiffness Non-Linearities," Int. J. Nonlinear Mech., 39, pp. 1079-1091.

[128] Gendelman, O. V., 2004, "Bifurcations of Nonlinear Normal Modes of Linea Oscillator With Strongly Nonlinear Damped Attachment," Nonlinear Dyn., 37, pp. 115-128.

[129] Pilipchuk, V. N., Vakakis, A. F., and Azeez, M. A. F., 1997, "Study of a Class of Subharmonic Motions Using a Non-Smooth Temporal Transformation," Phys. D., 100, pp. 145-164.

[130] Vakakis, A. F., McFarland, D. M., Bergman, L., Manevitch, L. I., and Gendelman, O., 2004, "Isolated Resonance Captures and Resonance Capture Cascades Leading to Single- or Multi-Mode Passive Energy Pumping in Damped Coupled Oscillators," ASME J. Vib. Acoust., 126(2), pp. 235-244.

[131] Starosvetsky, Y., and Gendelman, O. V., 2009, "Vibration Absorption in Systems Comprising Nonlinear Energy Sink: Nonlinear Damping," J. Sound Vib., 324, pp. 916-939.

[132] Gourdon, E., and Lamarque, C. H., 2005, "Energy Pumping for a Larger Span of Energy," J. Sound Vib., 285, pp. 711-720.

[133] Musienko, A. I., Lamarque, C. H., and Manevitch, L. I., 2006, "Design of Mechanical Energy Pumping Devices," J. Vib. Control, 12, pp. 355-371.

[134] Tsakirtzis, S., Panagopoulos, P. N., Kerschen, G., Gendelman, O., Vakakis, A. F., and Bergman, L. A., 2007, "Complex Dynamics and Targeted Energy Transfer in Systems of Linear Oscillators Coupled to Multi-Degrees-of-Freedom Essentially Nonlinear Attachments," Nonlinear Dyn., 48, pp. 285-318. 
[135] Vakakis, A. F., Manevitch, L. I., Gendelman, O., Bergman, L. A., 2003, "Dynamics of Linear Discrete Systems Connected to Local Essentially NonLinear Attachments," J. Sound Vib., 264, pp. 559-577.

[136] Tsakirtzis, S., Vakakis, A. F., and Panagopoulos, S., 2007, "Broadband Energy Exchanges Between a Dissipative Elastic Rod and a Multi-Degrees-of-Freedom Dissipative, Essentially Nonlinear Attachment,” Int. J. Nonlinear Mech., 42, pp. 36-57.

[137] Georgiades, F., and Vakakis, A. F., 2007, "Dynamics of a Linear Beam With an Attached Local Nonlinear Energy Sink," Commun Nonlinear Sci Numer Simul., 12, pp. 643-661.

[138] Panagopoulos, P. N., Georgiades, F., Tsakirtzis, S., Vakakis, A. F., and Bergman, L. A., 2007, "Multi-Scaled Analysis of the Damped Dynamics of an Elastic Continuum With an Essentially Nonlinear End Attachment," Int. J. Solids Struct., 44, pp. 6256-6278.

[139] Georgiades, F., and Vakakis, A. F., 2009, "Passive Targeted Energy Transfers and Strong Modal Interactions in the Dynamics of a Thin Plate With Strongly Nonlinear End Attachments," Int. J. Solids Struct., 46, pp. 2330-2353.

[140] Gendelman, O. V., Gourdon, E., and Lamarque, C. H., 2006, "Quasi-Periodic Energy Pumping in Coupled Oscillators Under Periodic Forcing," J. Sound Vib., 294, pp. 651-662.

[141] Gendelman, O. V., and Starosvetsky, Y., 2007, "Quasi-Periodic Response Regimes of Linear Oscillator Coupled to Nonlinear Energy Sink Under Periodic Forcing,” ASME J. Appl. Mech., 74(2), pp. 325-331.

[142] Gendelman, O. V., Starosvetsky, Y., and Feldman, M., 2008, "Attractors of Harmonically Forced Linear Oscillator With Attached Nonlinear Energy Sink I: Description of Response Regimes," Nonlinear Dyn., 51, pp. 31-46.

[143] Starosvetsky, Y., and Gendelman, O. V., 2008, "Strongly Modulated Response in Forced 2DOF Oscillatory System With Essential Mass and Potential Asymmetry," Phys. D., 237, pp. 1719-1733.
[144] Shiroky, I. B., and Gendelman, O. V., 2008, "Essentially Nonlinear Vibration Absorber in a Parametrically Excited System," ZAMM, 88, pp. 573-596.

[145] Sinha, S. C., Redkara, S., and Butcher, E. A., 2005, "Order Reduction of Nonlinear Systems with Time Periodic Coefficients Using Invariant Manifolds," J. Sound Vib., 284, pp. 985-1002.

[146] Gabale, A. P., and Sinha, S. C., 2011, "Model Reduction of Nonlinear Systems With External Periodic Excitations via Construction of Invariant Manifolds," J. Sound Vib., 330, pp. 2596-2607.

[147] Gabale, A., and Sinha, S. C., 2010, "Construction of Reduced Order Controllers for Nonlinear Systems With Periodic Coefficients," J. Vib. Control, 17, pp. 391-406.

[148] Steindl, A., and Troger, H., 2001, "Methods for Dimension Reduction and their Application in Nonlinear Dynamics," Int. J. Solids Struct., 38, pp. 2131-2147.

[149] Apiwattanalunggarn, P., Shaw, S., and Pierre, C., 2005, "Component Mode Synthesis Using Nonlinear Normal Modes," Nonlinear Dyn., 41, pp. 17-46.

[150] Rand, R. H., and Ramani, D. V., 2001, "Nonlinear Normal Modes in a System With Nonholonomic Constraints," Nonlinear Dyn., 25, pp. 49-64.

[151] Georgiou, I. T., and Schwartz, I. B., 2001, "Invariant Manifolds, Nonclassical Normal Modes, and Proper Orthogonal Modes in the Dynamics of the Flexible Spherical Pendulum," Nonlinear Dyn., 25, pp. 3-31.

[152] Jayaprakash, K. R., Starosvetsky, Y., Vakakis, A. F., Peeters, M., and Kerschen, G., 2011, "Nonlinear Normal Modes and Band Zones in Granular Chains With No Pre-Compression," Nonlinear Dyn., 63, pp. 359-385.

[153] Mikhlin, Y. V., and Volok, A. M., 2000, "Solitary Transversal Waves and Vibro-Impact Motions in Infinite Chains and Rods," Int. J. Solids Struct., 37(25), pp. 3403-3420.

[154] Burton, T. D., 2007, "Numerical Calculations of Nonlinear Normal Modes in Structural Systems," Nonlinear Dyn., 49, pp. 425-441.

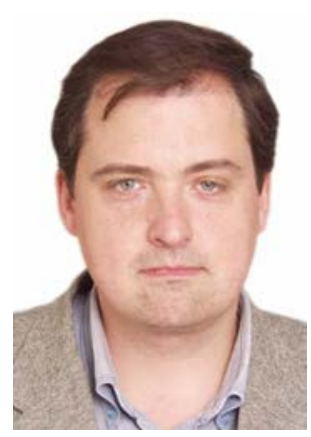

Konstantin Avramov is a Leading Researcher of the Department of Mechanical Vibrations at the Podgorny Institute for Mechanical Engineering Problems, National Academy of Sciences of Ukraine (Kharkov, Ukraine) and Professor of the Department of Gas and Fluid Mechanics at the National Technical University "Kharkov Polytechnic Institute." He is also Professor of Computer Science in Kharkov National University of Road Transport. Avramov received the Master in Dynamics and Strength of Machines (1991) from Kharkov Polytechnic Institute and a Ph.D. (1993) in Dynamics and Strength of Machines from Kharkov Polytechnic Institute and an Associate Professorship (1995) from the Department of Theoretical Mechanics of the Kharkov Polytechnic Institute. Avramov received a Doctor of Science in Mechanics of Solids (2004) from the Podgorny Institute for Mechanical Engineering Problems, National Academy of Sciences of Ukraine, and Full Professor (2005) from the Department of Gas and Fluid Mechanics in National Technical University "Kharkov Polytechnic Institute." His area of research includes nonlinear dynamics, asymptotic methods, stability and bifurcation theory, plates and shell theory, fluid structure interactions, dynamics of rotors and blades, and dynamics of rockets and satellites. He has published two books and over 300 papers in peer reviewed journals and proceedings. Avramov is organizer and Chair of the Mini-Symposiums at the European Nonlinear Oscillations Conferences (2008, 2011).

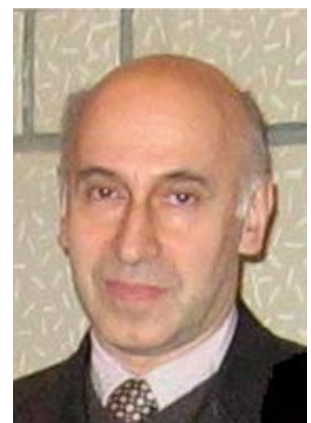

Yuri Mikhlin is Full Professor of the Department of Applied Mathematics at the National Technical University "Kharkov Polytechnic Institute." He graduated from the Dneptopetrovsk State University summa cum laude in Mechanics (1970); received a Ph.D. (Physics \& Mathematics) from Dnepropetrovsk State University (1974), and received a Doctor of Science (Physics \& Mathematics) from the Moscow Institute for Problems in Mechanics, Russian Academy of Sciences (1988). He was organizer and chairman of the Sci. Committees of Int. Conferences on Nonlinear Dynamics at the National Technical University "Kharkov Polytechnic Institute" (2004, 2007, 2010); he is also Organizer and Chair of the Mini-Symposiums at the European Nonlinear Oscillations Conferences $(2008,2011)$. His current research focuses on: nonlinear normal mode theory, nonlinear rotor dynamics, nonlinear dynamics of elastic systems, vibro-absorption problems, transient and localization problems, stability of motions, etc. He has published over 130 papers in peer reviewed journals and proceedings and five books. 